\title{
A THREE PHASE MODEL TO INVESTIGATE THE EFFECTS OF DEAD MATERIAL ON THE GROWTH OF AVASCULAR TUMOURS
}

\author{
Thomas D. Lewin ${ }^{1, *}$, Philip K. Maini ${ }^{1}$, Eduardo G. Moros ${ }^{2}$, \\ Heiko Enderling ${ }^{2,3}$ AND Helen M. Byrne ${ }^{2}$
}

\begin{abstract}
In vivo tumours are highly heterogeneous entities which often comprise intratumoural regions of hypoxia and widespread necrosis. In this paper, we develop a new three phase model of nutrient-limited, avascular tumour growth to investigate how dead material within the tumour may influence the tumour's growth dynamics. We model the tumour as a mixture of tumour cells, dead cellular material and extracellular fluid. The model equations are derived using mass and momentum balances for each phase along with appropriate constitutive equations. The tumour cells are viewed as a viscous fluid pressure, while the extracellular fluid phase is viewed as inviscid. The physical properties of the dead material are intermediate between those of the tumour cells and extracellular fluid, and are characterised by three key parameters. Through numerical simulation of the model equations, we reproduce spatial structures and dynamics typical of those associated with the growth of avascular tumour spheroids. We also characterise novel, non-monotonic behaviours which are driven by the internal dynamics of the dead material within the tumour. Investigations of the parameter sub-space describing the properties of the dead material reveal that the way in which non-viable tumour cells are modelled may significantly influence the qualitative tumour growth dynamics.
\end{abstract}

Mathematics Subject Classification. 92C50, 76T30.

Received November 15, 2018. Accepted September 2, 2019.

\section{INTRODUCTION}

Cancer is a complex family of diseases that typically emerges when successive genetic mutations accumulate over time, transforming a cell from a 'normal' to a malignant phenotype. However, regardless of disease type, many cancers share common traits that together promote the formation of a highly complex, heterogeneous tumour microenvironment $[17,18]$. In vivo, oxygen and nutrient supply are often insufficient to support the expansive and unregulated proliferation typical of tumour cell populations. This commonly leads to intratumoural regions of severe hypoxia and necrotic cell death, a feature not commonly observed in healthy, normal tissues $[6,31]$. The resulting cellular debris may constitute a significant proportion of the tumour volume and have a significant impact on its growth dynamics.

Keywords and phrases: avascular tumour growth, cell death, multiphase mixtures, moving boundaries.

1 Wolfson Centre for Mathematical Biology, Mathematical Institute, University of Oxford, Oxford, UK.

2 Radiation Oncology, H. Lee Moffitt Cancer Center \& Research Institute, Tampa, Florida, USA.

${ }^{3}$ Integrated Mathematical Oncology, H. Lee Moffitt Cancer Center \& Research Institute, Tampa, Florida, USA.

* Corresponding author: thomas.lewin@pmb.ox.ac.uk 
In addition to necrosis, several other mechanisms may contribute to cell death [28]. These include apoptosis, autophagy, senescence and mitotic catastrophe, among others. Furthermore, administering different anti-cancer therapies may induce different modes of cell death in different tumour regions [12]. Given this complexity in vivo, it is unclear how to model dying cells and dead material within the tumour and, further, how interactions of this material with viable cell populations may affect the tumour's net growth dynamics.

Avascular in vitro tumour spheroids represent a simplified, idealised environment in which to study aspects of tumour growth and treatment effects $[19,35]$. Oxygen, along with other nutrients required for proliferation, is supplied from the exterior of the tumour spheroid and is consumed by proliferating tumour cells. As in vitro tumour spheroids grow, the centre of the tumour becomes hypoxic and eventually develops into a necrotic core upon sustained nutrient deprivation. This central region of necrotic cell death counteracts the proliferation occurring at the spheroid boundary, resulting in deceleration of tumour growth towards a steady, equilibrium size. As such, tumour spheroids grown in this way typically have distinct, reproducible structures and follow dynamics which result in sigmoidal growth curves $[13,30]$.

The dynamics of avascular tumours have been modelled extensively. The simplest, spatially-resolved models of avascular tumour growth may be reduced to an ordinary differential equation (ODE) for the evolution of the external tumour boundary coupled to a reaction-diffusion equation for the intratumoural nutrient concentration [14]. Such models have been used to reproduce the tumour composition, nutrient consumption and growth saturation dynamics observed in vitro $[7,14-16,25]$.

More recently, more complex frameworks have been used to study the spatiotemporal dynamics of heterogeneous tumour growth. Multiphase models provide a continuum framework which may be used to investigate interactions between different components of a growing tissue, modelling the microenvironment as a mixture of two or more constituent phases. A range of models of this type have been used to model tumour growth, the simplest decomposing the tumour into two phases $[5,8,21,34]$. There are several different ways to model the material properties of each phase. Some models view the tumour as a mixture of fluid phases $[5,8]$, while others consider multiple phase types within the mixture to investigate the physical interactions and stresses induced within a growing tumour. These include rigid, non-deformable solid phases [23, 29], linear-elastic phases [2, 3] and those with viscoelastic/viscoplastic properties [1].

In this paper we develop a new three phase model to investigate the influence of dead material present within the tumour volume on its growth dynamics; we view the dead material as an 'intermediate' phase whose properties are intermediate between the viable tumour cell population and the extracellular fluid. We identify three key parameters in our model which summarise the mechanical properties of the dead material, with appropriate limits of these parameters corresponding to sub-cases of our model which are similar to previous two phase models in the literature. In Section 2 we develop our model and show how to reduce the system to a closed set of equations governing the dynamics of the tumour cell and dead material phases. In Section 3 we use numerical simulations to characterise the growth dynamics exhibited by the model, including some novel, nonmonotonic behaviours. In Section 4 we perform parameter sensitivity analyses to investigate how the properties of the dead material influence the tumour growth dynamics and thus the impact of accounting for dead cellular material within the tumour on model predictions.

\section{Model Development}

In this section we derive a new multiphase, mixture model of avascular tumour growth, extending existing two phase models which distinguish tumour cells and extracellular fluid [5, 8]. Our model proposes that a non-viable cell population may form a significant portion of the tumour and may affect its growth dynamics. We treat this phase as comprising of all of the non-viable cellular material and cellular debris within the tumour, irrespective of cell death mechanism or stage of decay. As such, this phase gives, in some sense, an 'averaged' description of this component of the tumour environment across all of these modes of cell death and is a transitional phase between the viable tumour population and the extracellular fluid. We hereafter refer to this phase simply as being comprised of 'dead material'. We view the dead material as a viscous fluid whose mechanical properties 
may differ from those of the tumour cells (also viewed as a viscous fluid) and extracellular fluid (viewed as inviscid).

The spatial distribution of each phase is described by the volume fractions $\phi_{i}(\mathbf{x}, t)(i=1,2,3)$ for the tumour cell, dead material and extracellular fluid phases, respectively. The movement of each phase is given by the phase velocities, $\mathbf{v}_{\mathbf{i}}(\mathbf{x}, t)$, with the phase pressures and stress tensors represented by $p_{i}(\mathbf{x}, t)$ and $\underline{\sigma}_{i}(\mathbf{x}, t)$, respectively. We apply mass and momentum balances to each phase to determine how the dependent variables evolve over time. Tumour growth is further assumed to depend on the availability of nutrients, in particular oxygen, and so the system is coupled to a reaction-diffusion equation for the oxygen concentration $c(\mathbf{x}, t)$. The governing equations are derived in detail in Sections 2.1-2.3.

\subsection{Growth model equations}

We apply conservation of mass and momentum to each phase $(i=1,2,3)$. We assume equal phase densities and justify this assumption by noting that the material comprising biological cells is broadly similar to the interstitial fluid. As such, mass fractions and volume fractions become equivalent and we may ignore densities in the resulting system of equations, which can be written as:

$$
\begin{gathered}
\frac{\partial \phi_{i}}{\partial t}+\nabla \cdot\left(\mathbf{v}_{\mathbf{i}} \phi_{i}\right)=S_{i}, \\
0=\nabla \cdot\left(\phi_{i} \underline{\boldsymbol{\sigma}}_{\boldsymbol{i}}\right)+\mathbf{F}_{\mathbf{i}} .
\end{gathered}
$$

We note that for biological tissues we consider slow-flow regimes and so the inertia terms can be neglected in the momentum equations at leading order [20, 27]. The model equations are specialised by specification of appropriate constitutive equations for the stress tensors, $\underline{\sigma}_{i}$, mass sources/sinks, $S_{i}$, and momentum sources/sinks, $\mathbf{F}_{\mathbf{i}}$; these are described in Section 2.2.

The governing equations are coupled to a reaction-diffusion equation for the oxygen field, $c$, given by

$$
0=D \nabla^{2} c-\Gamma \phi_{1} H_{\epsilon}\left(c-c_{N}\right)
$$

where the tumour cells consume oxygen at a rate $\Gamma$ and $D$ is the diffusion coefficient. We define

$$
H_{\epsilon}(c)=\frac{1}{2}\left(1+\tanh \left(\frac{c}{\epsilon}\right)\right)
$$

as a smooth approximation to the Heaviside function for oxygen consumption for small $\epsilon$. This term provides a smooth, monotonic description of oxygen consumption which saturates at large oxygen concentrations and goes to 0 at $c=0$. This term is similar to the form used by other authors [33]. The oxygen field is assumed to be in a quasi-steady state since the timescale for diffusion is much shorter than that for tumour growth (oxygen takes approximately 10 to diffuse $100 \mu \mathrm{m}$ whereas tumour doubling times are days or months [14]). This separation

of timescales enables us to neglect the dilution term that is required for reaction-diffusion equations on growing domains [10].

\subsection{Constitutive relations}

We close equations (2.1)-(2.3) by prescribing appropriate constitutive equations, along with initial and boundary conditions. Firstly, we assume that the mixture is saturated, that is, the three phases constitute the entirety of the tumour volume and so we impose the 'no voids' condition:

$$
\sum_{i=1}^{3} \phi_{i}=1 .
$$


The mass source and sink terms, $S_{i}$, in equation (2.1) account for mass transfer between the phases and may be associated with proliferation and cell death processes. The source term for the tumour cell phase, $S_{1}$, is given by

$$
S_{1}=\underbrace{\eta \phi_{1} \phi_{3} H_{\epsilon}\left(c-c_{H}\right)}_{\text {proliferation }}-\underbrace{\chi \phi_{1} H_{\epsilon}\left(c_{N}-c\right)}_{\text {necrosis }}-\underbrace{\kappa \phi_{1}}_{\text {apoptosis }} .
$$

The first term in equation (2.6) describes proliferation of the viable tumour cell population, with the rate of mitosis in normoxic conditions given by the parameter $\eta$. Proliferation is assumed to occur at a rate proportional to the product of the local volume fraction of the tumour cell phase, $\phi_{1}$, and the extracellular fluid, $\phi_{3}$, since the material and nutrients required for proliferation are assumed to be derived from this phase. We assume further that only well-oxygenated cells (i.e. those for which the local oxygen concentration exceeds the hypoxic threshold, $c_{H}$ ) may proliferate, with cells in regions where $c<c_{H}$ entering quiescence. The second term relates to necrosis induced by nutrient starvation when the oxygen concentration drops below the threshold $c_{N}<c_{H}$ and occurs at rate $\chi$. Proliferation and necrosis happens alongside a baseline level of apoptotic cell death which occurs throughout the tumour at a constant rate, $\kappa$ (the third term).

Material lost from the tumour phase due to cell death acts as a source term in the mass balance equation for the dead material phase (Eq. (2.7)). If we assume that the dead material is degraded at a constant rate, $\lambda$, and proportional to the number of dead cells, then this phase undergoes exponential decay and we may write

$$
S_{2}=\chi \phi_{1} H_{\epsilon}\left(c_{N}-c\right)+\kappa \phi_{1}-\underbrace{\lambda \phi_{2}}_{\text {cellular decay }}
$$

Our three phase mixture is assumed to conserve mass locally, with volume changes due to cell proliferation and death occurring as a result of mass transfer between the phases, and so we impose

$$
S_{3}=-\left(S_{1}+S_{2}\right)=\lambda \phi_{2}-\eta \phi_{1} \phi_{3} H_{\epsilon}\left(c-c_{H}\right) .
$$

As such, the fluid phase is required for local proliferation in the cell phase, while the dead material decays to become part of the extracellular fluid.

In prescribing the momentum source/sink terms, $\mathbf{F}_{\mathbf{i}}$, in equation (2.2) we follow the form typically used in the literature [20] and impose

$$
\mathbf{F}_{\mathbf{i}}=p_{i} \nabla \phi_{i}+\sum_{\substack{j=1 \\ j \neq i}}^{3} d_{i j} \phi_{j} \phi_{i}\left(\mathbf{v}_{\mathbf{j}}-\mathbf{v}_{\mathbf{i}}\right)
$$

The first term in equation (2.9) describes how the phase pressure $p_{i}$ drives transport down gradients in the volume fraction of phase $i$. We note that there is no consensus for the pressure dependence in this term; some authors prefer to use the global pressure $p[5,8]$, while others use the phase pressures $p_{i}[20]$. In both cases, this term satisfies a Lagrangian multiplier constraint related to the no voids condition (Eq. (2.5)) since the phase pressures, $p_{i}$, include a term for the global pressure, $p$ (see Eq. (2.11)). Simulations instead specifying $p \nabla \phi_{i}$ in this term revealed a similar range of qualitative behaviours (results not shown), as might be expected due to the isotropic nature of the stress tensors, $\underline{\sigma}_{i}$ (see Eq. (2.10)). As such, the results in this paper are not significantly affected by this choice.

The second term in equation (2.9) describes the momentum exchange, or drag, due to the differential movement of the constituent phases. The drag terms are assumed to sum to 0 over all phases so the interphase drag coefficients satisfy $d_{i j}=d_{j i}$. 


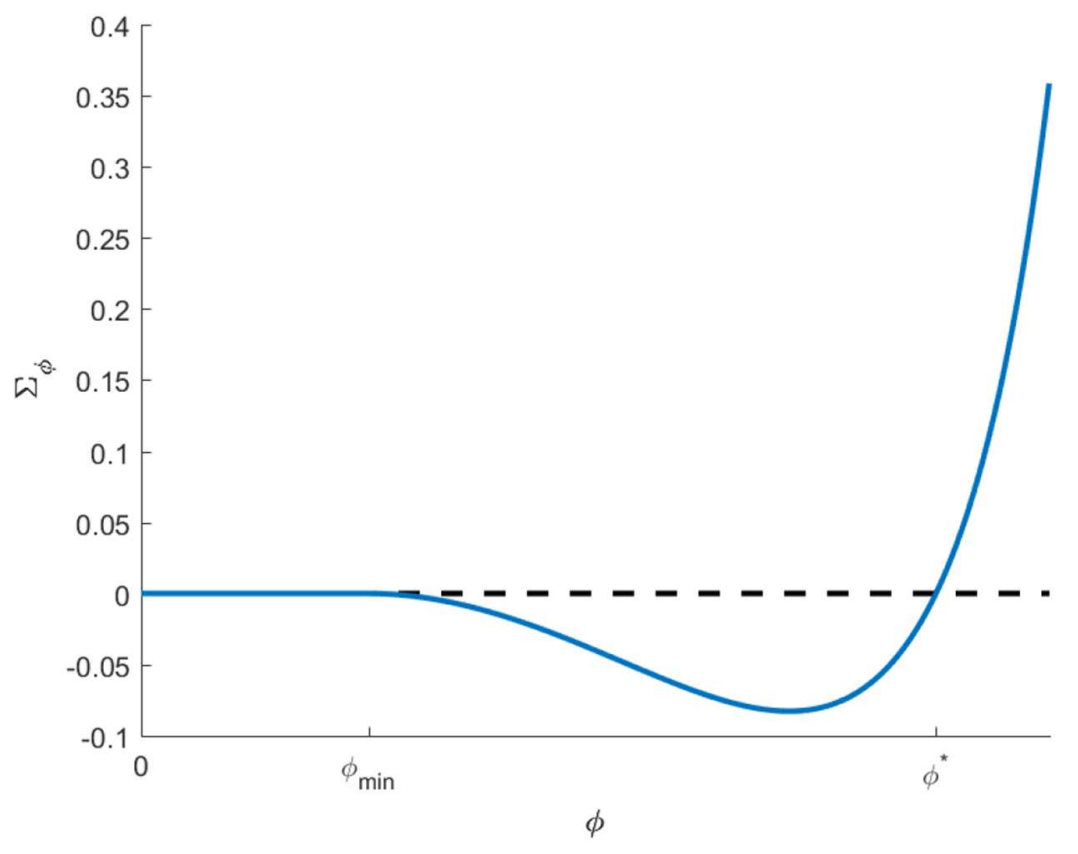

FiguRE 1. Illustrative sketch showing the dependence of the cell-sensing pressure $\Sigma_{\phi}(\phi)$ on the cell volume fraction $\phi$ (defined by Eq. (2.12)). We note that there is no interaction between cells if they are too sparsely seeded $\left(\Sigma_{\phi}=0\right.$ if $\left.\phi \leq \phi_{\min }\right)$. For intermediate volume fractions the cells tend to aggregate $\left(\Sigma_{\phi}<0\right.$ for $\left.\phi \in\left(\phi_{\min }, \phi^{*}\right)\right)$, whereas at high volume fractions the cells repel each other $\left(\Sigma_{\phi}>0\right.$ for $\left.\phi \in\left(\phi^{*}, 1\right)\right)$.

The stress tensors, $\underline{\boldsymbol{\sigma}}_{\boldsymbol{i}}$, account for the mechanical properties of each phase. We treat each component of the mixture as a fluid phase with potentially different viscosities and so the $\underline{\sigma}_{i}$ take the form given in equation (2.10) (I denotes the unit tensor). In particular we specify an ordering of the phases in which the dead material phase has intermediate viscosity, $\mu_{2}=\theta_{\mu} \mu_{1}$, between the viscous tumour phase, with viscosity $\mu_{1}$, and the inviscid extracellular fluid, where $0 \leq \theta_{\mu} \leq 1$. We note that while the mixture as a whole is incompressible (due to Eq. (2.5)), the same is not true for each phase due to the local effects of cell proliferation and so we retain the bulk viscosity term, $\lambda_{i}$. However we follow Hubbard et al. [20], and assume local thermodynamic equilibrium in which case $\lambda_{i}=-\frac{2}{3} \mu_{i}$.

$$
\underline{\boldsymbol{\sigma}}_{i}=\underbrace{-p_{i} \mathbf{I}}_{\text {isotropic phase pressure }}+\underbrace{\mu_{i}\left(\nabla \mathbf{v}_{\mathbf{i}}+\nabla \mathbf{v}_{\mathbf{i}}^{T}\right)}_{\text {shear viscosity }}+\underbrace{\lambda_{i}\left(\nabla \cdot \mathbf{v}_{\mathbf{i}}\right) \underline{\mathbf{I}}}_{\text {bulk viscosity }}, \quad \underbrace{\lambda_{i}=-\frac{2}{3} \mu_{i}}_{\text {thermodynamic equilibrium }}, \quad \underbrace{\mu_{1} \geq \mu_{2}=\theta_{\mu} \mu_{1} \geq \mu_{3}=0 .}_{\text {relative ordering of viscosities }}
$$

The momentum interaction terms, $\mathbf{F}_{\mathbf{i}}$, in equation (2.9) and the stress tensors, $\underline{\boldsymbol{\sigma}}_{\boldsymbol{i}}$, in equation (2.10) include phase-specific pressure terms, $p_{i}$, which need to be specified to close the system. Following [8] we assume a global isotropic fluid pressure, $p$, with an additional pressure, $\Sigma_{\phi}$, representing cell-sensing ascribed to the cell and dead material phases. The cell-sensing capacity of the dead material is assumed to be intermediate between that of the tumour cells and the extracellular fluid, which we model via the parameters $0 \leq \theta_{p}, \theta_{\mu} \leq 1$. A positive value of $\Sigma_{\phi}$ corresponds to a repulsive cell pressure, whilst negative values correspond to regions of cellular adhesion (see Fig. 1). The phase pressures, $p_{i}$, are specified in equation (2.11), while the cell-sensing term, $\Sigma_{\phi}$, 
is defined in equation (2.12) where $H(\cdot)$ is the Heaviside function:

$$
\begin{gathered}
p_{1}=p+\Sigma_{\phi}\left(\phi_{1}+\theta_{\Sigma} \phi_{2}\right), \quad p_{2}=p+\theta_{p} \Sigma_{\phi}\left(\phi_{1}+\theta_{\Sigma} \phi_{2}\right), \quad p_{3}=p \\
\Sigma_{\phi}(\phi)=\frac{\zeta\left(\phi-\phi_{\min }\right)^{2}\left(\phi-\phi^{*}\right)}{(1-\phi)} H\left(\phi-\phi_{\min }\right)
\end{gathered}
$$

Of particular note is the manner in which we model the dead material phase, which has volume fraction $\phi_{2}$. As stated previously, we view the dead material as a viscous fluid whose properties are intermediate between those of the tumour cells and the extracellular fluid phases. One of the main aims of this work is to investigate how the way in which the physical properties of the dead material are modelled affects the system dynamics. The parameter vector $\boldsymbol{\theta}=\left(\theta_{\mu}, \theta_{p}, \theta_{\Sigma}\right)$ summarises the mechanical properties of this phase relative to the other two phases in the mixture, with $\boldsymbol{\theta}$ taking values in $[0,1]^{3}$. In particular, from equations $(2.10)-(2.12)$ we note that $\boldsymbol{\theta}=(1,1,1)$ corresponds to a two-phase sub-case of the full model in which the dead material is essentially a compartment of the cellular phase and retains the same mechanical properties as the viable tumour cells. Conversely, the limit $\boldsymbol{\theta}=(0,0,0)$ pertains to a two-phase model in which the dead phase has the same properties as the extracellular fluid.

The parameter $\theta_{\mu}$ specifies the viscosity of the dead phase relative to the cell phase (see Eq. (2.10)). The parameters $\theta_{p}$ and $\theta_{\Sigma}$ pertain to relative cell-sensing function of this phase. Biologically, this cell-sensing may arise through inter-cellular interactions between cells and their filopodia in the case of cellular adhesion, or stress imposed on the cell membrane and cytoskeleton in regions of high cellular density, for example. Depending on the cell death pathway and stage of decay, we propose that cells in this phase may retain some of this function. In particular, $\theta_{p}$ characterises the relative ability of the non-viable cell population to perform this type of activity, while the parameter $\theta_{\Sigma}$ represents the relative contribution of this phase to the cell-sensing pressures experienced by the surrounding cells, as defined in equation (2.11).

A summary of all the model parameters defined in the system of equations (2.1)-(2.12) is given in Table 1.

\subsection{Initial and boundary conditions}

Equations (2.1)-(2.12) govern the dynamics of our multiphase model. When closed with appropriate initial and boundary conditions, this model describes the spatial and temporal evolution of a tumour growing in 3D. For simplicity, in this paper we hereafter restrict attention to a 1D Cartesian geometry. We assume symmetry in our growing tumour and define coordinates such that $x=0$ is at the centre of the tumour, where we impose symmetry conditions for the phase velocities, $v_{i}$, and oxygen concentration, $c$ :

$$
v_{i}=0, \quad \frac{\partial c}{\partial x}=0 \quad \text { at } \quad x=0
$$

The tumour boundary at $x=R(t)$ is defined to be at the exterior of the cell phase where the volume fraction $\phi_{1}$ drops to 0 . The boundary then moves with the tumour cell phase velocity here such that

$$
\frac{\mathrm{d} R}{\mathrm{~d} t}=\left.v_{1}\right|_{x=R(t)} .
$$

We impose Dirichlet boundary conditions for the isotropic pressure, $p$, and the oxygen concentration, $c$, at the exterior boundary:

$$
p=0, \quad c=c_{\infty} \quad \text { at } \quad x=R(t) .
$$

The tumour is assumed to grow into an extracellular fluid which offers little (or no) resistance to movement. In this scenario we assume that the boundary of the tumour is stress-free. Additionally, following Tao and 
TABLE 1. Table of multiphase model parameters appearing in equations (2.1)-(2.12).

\begin{tabular}{cl}
\hline Symbol & Parameter \\
\hline$\Gamma$ & $\mathrm{O}_{2}$ consumption rate \\
$D$ & $\mathrm{O}_{2}$ diffusion coefficient \\
$c_{\infty}$ & Normoxic $\mathrm{O}_{2}$ concentration at exterior boundary \\
$c_{H}$ & Tumour hypoxia threshold \\
$c_{N}$ & Tumour necrosis threshold \\
\hline$\epsilon$ & Heaviside smoothing parameter \\
\hline$\eta$ & Proliferation rate \\
$\kappa$ & Apoptosis rate \\
$\chi$ & Necrosis rate \\
$\lambda$ & Dead material decay rate \\
\hline$d_{i j}$ & Drag coefficient of phase $j$ on phase $i$ \\
$\mu_{i}$ & Viscosity of phase $i$ \\
\hline$\theta_{\mu}$ & Relative viscosity of dead and tumour phases \\
$\theta_{p}$ & Relative 'cell-sensing' ability of dead phase \\
$\theta_{\Sigma}$ & Relative influence of dead phase on cell-sensing \\
\hline$\zeta$ & Cell-sensing strength \\
$\phi^{*}$ & 'Natural' cell volume fraction \\
$\phi_{\min }$ & Minimum cell-sensing volume fraction \\
\hline
\end{tabular}

Rajagopal [26, 32], we assume proportional splitting of traction on this boundary for our other boundary condition $^{1}$ which gives

$$
\frac{4}{3} \mu_{1} \frac{\partial v_{1}}{\partial x}-\Sigma_{\phi}\left(\phi_{1}+\theta_{\Sigma} \phi_{2}\right)=\frac{4}{3} \theta_{\mu} \mu_{1} \frac{\partial v_{2}}{\partial x}-\theta_{p} \Sigma_{\phi}\left(\phi_{1}+\theta_{\Sigma} \phi_{2}\right)=0 \quad \text { at } \quad x=R(t) .
$$

Equations (2.13) and (2.16) thus specify two boundary conditions for each of the phase velocities $v_{1}$ and $v_{2}$ whose evolution is described by two coupled second order elliptic equations (Eq. (2.2)).

Equation (2.1) is a hyperbolic equation for the evolution of the volume fraction $\phi_{1}$. Since the exterior boundary moves with the phase velocity $v_{1}$, we have that both $x=R(t)$ and $x=0$ are characteristics and so we require no additional boundary conditions for the volume fraction $\phi_{1}$. This is not the case for the volume fraction $\phi_{2}$. Where the exterior boundary of the tumour is an outflow boundary for this phase $\left(v_{2}>v_{1}\right.$ at $\left.x=R(t)\right)$ then the solution for $\phi_{2}$ may still be specified along characteristics and we do not need to specify any additional boundary condition on $x=R(t)$. However, when we have an inflow boundary we must impose an additional boundary condition for this phase. We assume that any cellular debris that flows across the tumour boundary is cleared or decays away such that we have an exterior boundary layer for this phase in which the volume fraction $\phi_{2}$ drops to 0 , allowing for no inflow of non-viable material into the tumour. As such, we impose

$$
\phi_{2}^{+}=0 \quad \text { at } \quad x=R(t),
$$

where $\phi_{2}^{+}$represents the volume fraction of this phase immediately exterior to the tumour boundary $R(t)$.

It then only remains to specify the initial conditions:

$$
\phi_{1}=\tilde{\phi}_{1}(x), \quad \phi_{2}=\tilde{\phi}_{2}(x), \quad R=R_{0} \quad \text { at } \quad t=0,
$$

\footnotetext{
${ }^{1}$ We note that these boundary conditions do not require a factor of $\phi_{1}$ and $\phi_{2}$, respectively, since in our simulations we use non-zero initial conditions and thus the source terms, $S_{1}$ and $S_{2}$, in equations (2.6) and (2.7) ensure that $\phi_{1}$ and $\phi_{2}$ remain non-zero.
} 
where $\tilde{\phi}_{1}(x)$ and $\tilde{\phi}_{2}(x)$ are the initial volume fractions of the tumour cell and dead material phases, respectively, and $R_{0}$ is the initial outer tumour radius.

\subsection{Reducing the full multiphase system}

Equations (2.1)-(2.18) describe the dynamics of our multiphase mixture. We may simplify this system by eliminating the volume fraction and velocity pertaining to the extracellular fluid, $\phi_{3}$ and $v_{3}$, and the isotropic global pressure, $p$.

The no voids condition given by equation (2.5) gives

$$
\phi_{3}=1-\phi_{1}-\phi_{2}
$$

By summing the equations for conservation of mass of each phase (Eq. (2.1)), integrating with respect to $x$ and applying the boundary conditions at $x=0$, we obtain an identity for the mixture velocity, $\sum_{i} \phi_{i} v_{i}=0$. As such, the velocity of the fluid phase is given by

$$
v_{3}=-\frac{1}{\phi_{3}}\left(\phi_{1} v_{1}+\phi_{2} v_{2}\right) .
$$

Similarly, by summing the equations for conservation of momentum (Eq. (2.2)), we may obtain an expression for $\frac{\partial p}{\partial x}$ in terms of the volume fractions $\phi_{1}$ and $\phi_{2}$, and the velocities $v_{1}$ and $v_{2}$ :

$$
\frac{\partial p}{\partial x}=-\phi_{1} \frac{\partial \Sigma_{\phi}}{\partial x}-\theta_{p} \phi_{2} \frac{\partial \Sigma_{\phi}}{\partial x}+\frac{4}{3} \mu_{1} \frac{\partial}{\partial x}\left(\phi_{1} \frac{\partial v_{1}}{\partial x}\right)+\frac{4}{3} \theta_{\mu} \mu_{1} \frac{\partial}{\partial x}\left(\phi_{2} \frac{\partial v_{2}}{\partial x}\right)
$$

In this way, we obtain a reduced system of equations describing the evolution of the volume fractions $\phi_{1}$ and $\phi_{2}$, the phase velocities $v_{1}$ and $v_{2}$, and the oxygen tension $c$. The eliminated variables $\phi_{3}, v_{3}$ and $p$ may be recovered from equations $(2.19)-(2.21)$.

\subsection{Summary of reduced model equations}

For completeness, we state below the reduced system of equations that define our multiphase model of tumour growth. A table summarising all of the parameters that appear in the governing equations is presented in Table 1.

\section{Mass conservation equations:}

$$
\begin{aligned}
& \frac{\partial \phi_{1}}{\partial t}+\frac{\partial}{\partial x}\left(\phi_{1} v_{1}\right)=\eta \phi_{1}\left(1-\phi_{1}-\phi_{2}\right) H_{\epsilon}\left(c-c_{H}\right)-\chi \phi_{1} H_{\epsilon}\left(c_{N}-c\right)-\kappa \phi_{1}, \\
& \frac{\partial \phi_{2}}{\partial t}+\frac{\partial}{\partial x}\left(\phi_{2} v_{2}\right)=\chi \phi_{1} H_{\epsilon}\left(c_{N}-c\right)+\kappa \phi_{1}-\lambda \phi_{2},
\end{aligned}
$$

Oxygen profile:

$$
0=D \frac{\partial^{2} c}{\partial x^{2}}-\Gamma \phi_{1} H_{\epsilon}\left(c-c_{H}\right)
$$

\section{Momentum balances:}

$$
\begin{aligned}
0= & \frac{4}{3} \mu_{1}\left(1-\phi_{1}\right) \frac{\partial}{\partial x}\left(\phi_{1} \frac{\partial v_{1}}{\partial x}\right)-\frac{4}{3} \theta_{\mu} \mu_{1} \phi_{1} \frac{\partial}{\partial x}\left(\phi_{2} \frac{\partial v_{2}}{\partial x}\right)+d_{12} \phi_{1} \phi_{2}\left(v_{2}-v_{1}\right) \\
& -d_{13} \phi_{1}\left(\phi_{2} v_{2}+\left(1-\phi_{2}\right) v_{1}\right)-\phi_{1}\left(1-\phi_{1}\right) \frac{\partial \Sigma_{\phi}}{\partial x}+\theta_{p} \phi_{1} \phi_{2} \frac{\partial \Sigma_{\phi}}{\partial x}
\end{aligned}
$$




$$
\begin{gathered}
0=-\frac{4}{3} \mu_{1} \phi_{2} \frac{\partial}{\partial x}\left(\phi_{1} \frac{\partial v_{1}}{\partial x}\right)+\frac{4}{3} \theta_{\mu} \mu_{1}\left(1-\phi_{2}\right) \frac{\partial}{\partial x}\left(\phi_{2} \frac{\partial v_{2}}{\partial x}\right)+d_{12} \phi_{1} \phi_{2}\left(v_{1}-v_{2}\right) \\
-d_{23} \phi_{2}\left(\phi_{1} v_{1}+\left(1-\phi_{1}\right) v_{2}\right)-\theta_{p} \phi_{2}\left(1-\phi_{2}\right) \frac{\partial \Sigma_{\phi}}{\partial x}+\phi_{1} \phi_{2} \frac{\partial \Sigma_{\phi}}{\partial x} \\
\Sigma_{\phi}(\phi)=\frac{\zeta\left(\phi-\phi_{\min }\right)^{2}\left(\phi-\phi^{*}\right)}{(1-\phi)} H\left(\phi-\phi_{\min }\right)
\end{gathered}
$$

where

\section{Initial and boundary conditions:}

$$
\begin{gathered}
\frac{\mathrm{d} R}{\mathrm{~d} t}=\left.v_{1}\right|_{x=R(t)}, \\
\frac{\partial c}{\partial x}=0, \quad v_{1}=v_{2}=0 \quad \text { at } \quad x=0, \\
\phi_{2}^{+}=0, \quad c=c_{\infty} \quad \text { at } \quad x=R(t), \\
\frac{4}{3} \mu_{1} \frac{\partial v_{1}}{\partial x}-\Sigma_{\phi}\left(\phi_{1}+\theta_{\Sigma} \phi_{2}\right)=\frac{4}{3} \theta_{\mu} \mu_{1} \frac{\partial v_{2}}{\partial x}-\theta_{p} \Sigma_{\phi}\left(\phi_{1}+\theta_{\Sigma} \phi_{2}\right)=0 \quad \text { at } \quad x=R(t), \\
\phi_{1}=\tilde{\phi}_{1}(x), \quad \phi_{2}=\tilde{\phi}_{2}(x), \quad R=R_{0} \quad \text { at } \quad t=0 .
\end{gathered}
$$

\subsection{Numerical solution of the model equations}

We solve equations (2.22)-(2.32) numerically in order to simulate tumour growth for a variety of parameter regimes. We develop a simple, stable finite difference scheme, however we note that a more sophisticated scheme may be required for detailed investigation of certain cases. A description of the numerical method is presented in Appendix A.

For all simulations, we start with an initial radius $R_{0}=0.3$. The initial volume fraction of tumour cells is uniform on $x=\left[0, R_{0}\right]$ with volume fraction $\tilde{\phi}_{1}=\phi^{*}$. Since the governing equations are singular when $\phi_{2} \equiv 0$, we prescribe the uniform initial condition $\tilde{\phi}_{2}=0.005$ for $x=\left[0, R_{0}\right]$.

The choice of uniform initial composition does not significantly affect the resulting evolution of the tumour over time (results not shown). Varying the uniform initial volume fraction of the tumour cell phase, $\tilde{\phi}_{1}$, simply introduces a small time delay during which the material within the tumour is redistributed before starting on the same growth trajectory. More specifically, if $\tilde{\phi}_{1}+\theta_{\Sigma} \tilde{\phi}_{2}<\phi^{*}$ on $x=\left[0, R_{0}\right]$ then the adhesion in the tumour phase, as given by $\Sigma_{\phi}$ in equation (2.12), results in an initial contraction of the tumour radius while the volume fraction of the tumour phase within the tumour increases. When $\left.\Sigma_{\phi}\left(\phi_{1}+\theta_{\Sigma} \phi_{2}\right)\right|_{x=R(t)}>0$, the tumour radius starts to increase and the tumour follows the same qualitative growth trajectory as for other choices of initial condition. Similar behaviour was reported by Breward et al. [5] for a two phase model of avascular tumour growth.

In a similar manner, we may vary the initial tumour radius, $R_{0}$. For all values of $R_{0}$ chosen, our simulations resulted in the same steady state configuration (results not shown). However for simulations in which the internal composition is important in driving the qualitative growth behaviour (cf. Sects. 3.3 and 3.4), starting the simulation at too large an initial radius may result in a qualitatively different trajectory moving towards the steady state. In these cases, the uniform initial tumour composition, $\tilde{\phi}_{1}$, does not have enough time to be redistributed in order to influence the dynamics in the manners described in Sections 3.3 and 3.4. However for all reasonable, small values of $R_{0}$ relative to the steady state tumour radius, the resulting trajectories simulated were the same. 
TABLE 2. The parameter ranges included in the parameter sweep for the multiphase tumour growth model as defined by equations $(2.22)-(2.32)$.

\begin{tabular}{ll}
\hline Parameter & Values for parameter sweep \\
\hline$c_{N}$ & $\{0.1,0.2,0.3\}$ \\
$\eta$ & $\{0.5,1,1.5,3\}$ \\
$\chi$ & $\{0.1,0.3,0.5,0.75,1,2\}$ \\
$\kappa$ & $\{0.02,0.05,0.1,0.5\}$ \\
$\lambda$ & $\{0.05,0.1,0.15,0.25,0.5,0.75\}$ \\
$d_{13}=d_{23}$ & $\{0.5,1,2,3\}$ \\
$\zeta^{*}$ & $\{0.5,0.6,0.7,0.8\}$ \\
$\phi^{*}$ & $\{0,0.2,0.5,0.75,1\} \times \phi^{*}$ \\
$\phi_{\min }$ & $\{0.5,1,2,3,5\}$ \\
$\mu_{1}$ & $\{0.1,0.25,0.5,0.75,1\}$ \\
$\theta_{\mu}$ & $\{0.1,0.25,0.5,0.75,1\}$ \\
$\theta_{p}$ & $\{0.1,0.25,0.5,0.75,1\}$ \\
$\theta_{\Sigma}$ &
\end{tabular}

TABLE 3. Table of fixed model parameter values for all simulations.

\begin{tabular}{cc}
\hline Parameter & Value \\
\hline$\Gamma$ & 2 \\
$c_{\infty}$ & 1 \\
$c_{H}$ & 0.3 \\
$D$ & 1 \\
$d_{12}$ & 1 \\
\hline
\end{tabular}

\section{Characterising tumour growth Dynamics}

Typical values for many of the model parameters are unclear. As such, we may rescale the variables in our model by typical values and hereafter consider all parameters as dimensionless quantities. We investigate the dynamics of growing tumours under these model equations by performing a sweep over a range of parameter values and characterising the qualitative growth behaviours observed. We vary 13 different model parameters, selecting parameter values from the sets given in Table 2. The remaining model parameters are held fixed at the values stated in Table 3 . We simulate the model for each parameter combination up to time $t=100$.

We characterise the qualitative dynamics identified by this parameter sweep in Sections 3.1-3.4. In Section 3.1 we describe monotonic tumour growth which approaches a steady-state, while in Section 3.2 we present typical travelling wave solutions. The qualitative behaviours described in Sections 3.1 and 3.2 are consistent with experimental observations, the resulting spatial structures reproducing those of avascular spheroids. Such results have been reported previously for similar (and also simpler) models [5, 8]. In Sections 3.3 and 3.4 we characterise two types of non-monotonic dynamics exhibited by our multiphase model that, to the best of our knowledge, have not been reported for existing models of avascular tumour growth. The parameter values for each of the simulation results discussed in this paper are given in Table 4. In Section 3.5 we catalogue the parameter sets from our sweep for which the tumour approaches a steady state size.

\subsection{Steady state solutions}

The typical evolution of a tumour spheroid follows monotonic, sigmoidal growth towards a steady state [13]. Our model reproduces these dynamics and one such example is presented in Figure 2. 
TABLE 4. Table of parameter values for each simulation discussed; cases i-iv pertain to simulation results presented in Section 3, whereas cases A-D refer to simulation results discussed in Section 4.

\begin{tabular}{lccccccccccccc}
\hline & \multicolumn{110}{c}{ Parameter value } \\
\cline { 2 - 15 } Case & $c_{N}$ & $\eta$ & $\chi$ & $\kappa$ & $\lambda$ & $d_{13}=d_{23}$ & $\zeta$ & $\phi^{*}$ & $\phi_{\min }$ & $\mu_{1}$ & $\theta_{\mu}$ & $\theta_{p}$ & $\theta_{\Sigma}$ \\
\hline i & 0.1 & 1 & 1 & 0.05 & 0.1 & 0.1 & 3 & 0.8 & 0 & 1 & 0.5 & 1 & 1 \\
ii & 0.1 & 2 & 1 & 0.05 & 0.2 & 1 & 2 & 0.6 & 0.12 & 3 & 0.25 & 1 & 0.5 \\
iii & 0.2 & 1 & 1 & 0.05 & 0.05 & 0 & 2 & 0.8 & 0.16 & 0.5 & 0.1 & 0.5 & 1 \\
iv & 0.3 & 0.5 & 0.75 & 0.02 & 0.5 & 0 & 2 & 0.7 & 0 & 2 & 0.75 & 0.25 & 0.1 \\
A & 0.1 & 0.5 & 0.1 & 0.02 & 0.5 & 0 & 3 & 0.8 & 0 & 5 & 0.75 & 0.25 & 1 \\
B & 0.1 & 0.5 & 2 & 0.05 & 0.5 & 0.1 & 0.5 & 0.8 & 0.16 & 0.5 & 0.25 & 0.1 & 0.5 \\
C & 0.1 & 1 & 0.75 & 0.1 & 0.05 & 0.5 & 3 & 0.6 & 0.45 & 2 & 0.1 & 0.25 & 0.25 \\
D & 0.1 & 0.5 & 0.3 & 0.05 & 0.1 & 0.1 & 2 & 0.8 & 0.16 & 2 & 1 & 0.1 & 1 \\
\hline
\end{tabular}
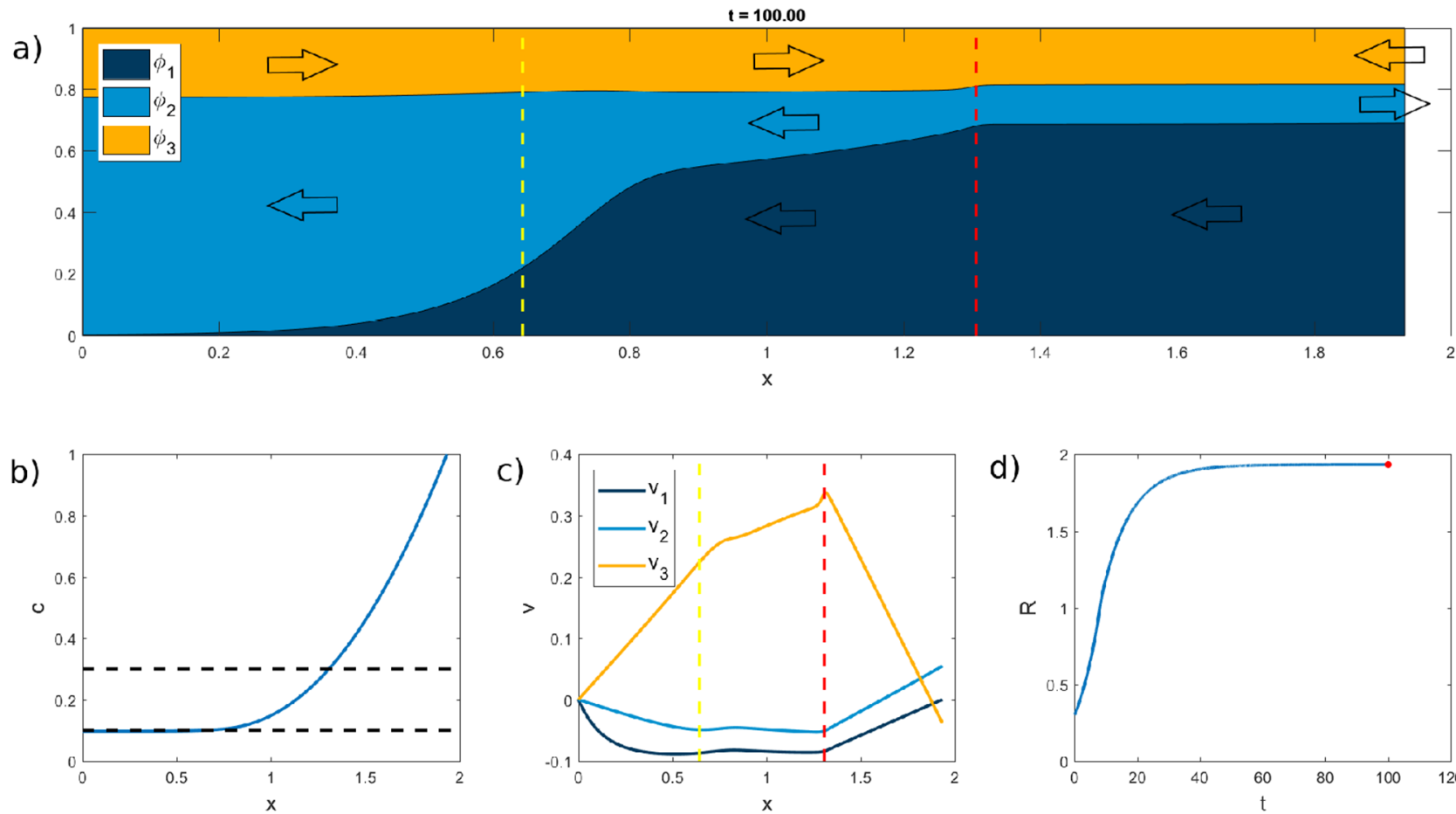

C)

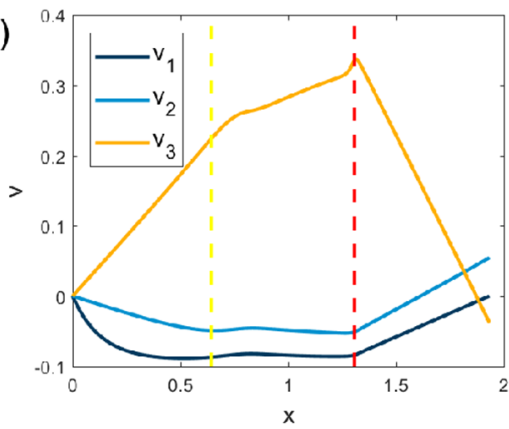

d)

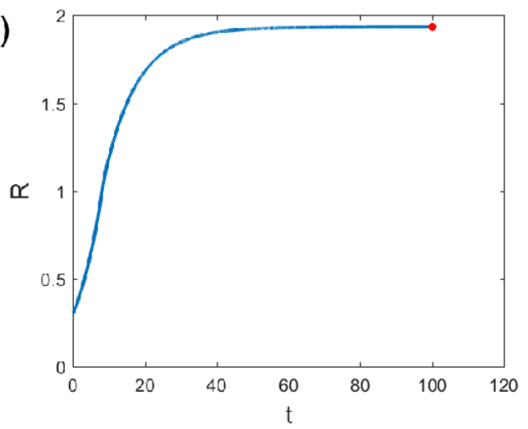

FigURE 2. Example of a simulation in which the tumour grows monotonically and approaches a steady state, solving equations (2.22)-(2.32) and using the parameter values in Tables 3 and 4 , case i. (a) A cross-section of tumour profile at time $t=100$ showing the internal distribution of each phase. The volume fraction of the tumour cells, $\phi_{1}$, is shown in dark blue, the dead material volume fraction, $\phi_{2}$, is shown in light blue, and the volume fraction for the extracellular fluid, $\phi_{3}$, is represented in yellow. The dashed red and yellow vertical lines mark the positions of the internal radii $R_{H}$ and $R_{N}$, respectively. The arrows represent the internal flow of material at steady state. (b) The oxygen concentration, $c$, throughout the tumour at time $t=100$ (blue line). The black horizontal dashed lines mark the thresholds for hypoxia and necrosis $\left(c_{H}>c_{N}\right)$. (c) The phase velocities $v_{i}$ for $i=1,2,3$ at time $t=100$ coloured corresponding to the respective volume fractions, $\phi_{i}$. (d) The tumour radius trajectory $R(t)$ (blue line) with the red point marking the time point $t=100$ corresponding the other plots. 


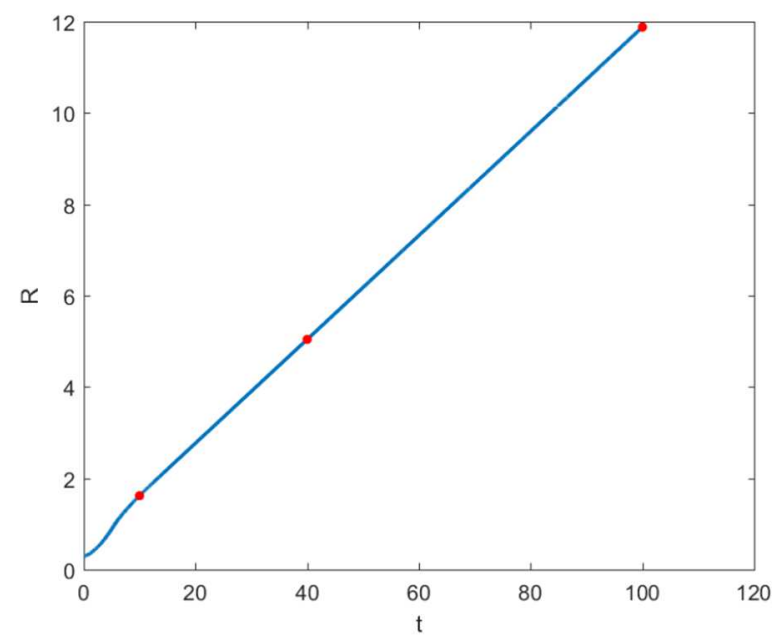

(A)
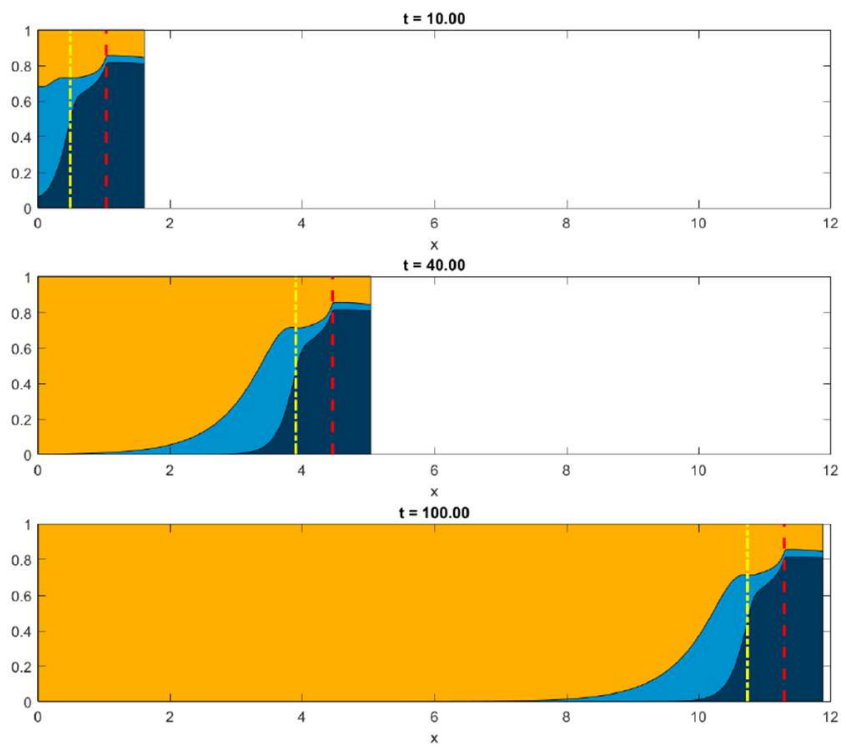

(B)

Figure 3. (a) Tumour radius trajectory (blue curve) exhibiting travelling wave behaviour from simulation of equations (2.22)-(2.32) using the parameter values in Tables 3 and 4 , case ii. The red dots mark the time points $t=10,40,100$ for which we visualise the tumour composition. (b) Visualisation of the internal composition of the tumour for the time series $t=10,40,100$ showing the showing how the tumour's spatial structure changes over time as it evolves to a travelling wave. The composition of the tumour is represented as in Figure 2.

Figure 2 visualises a snapshot of the tumour at the end of the simulation at $t=100$. We observe an outer proliferative rim, $R_{H}<x<R$, in which the tumour phase volume fraction is approximately constant. At $x=$ $R_{H}=1.31$, the oxygen concentration, which decreases monotonically with distance from the tumour boundary, attains the threshold value for hypoxia, $c_{H}$, and a quiescent region forms for $x<R_{H}$. At $x=R_{N}=0.65$, the oxygen concentration reaches the threshold for necrosis, $c_{N}$, and we observe the formation of a central necrotic core, characterised by a large volume fraction of dead material, $\phi_{2}$, and a low value of the tumour cell volume fraction, $\phi_{1}$.

We note that when the steady state is achieved the tumour is not in a dormant state: equilibrium arises when the mass gained in the outer, well-oxygenated region of the tumour by cell proliferation balances that lost in the central necrotic core due to cell death. The tumour phase velocity, $v_{1}$, is zero at the tumour boundary, $v_{1}(x, t)<0$ for $x \in(0, R)$. As such, an internal circulation is set up whereby proliferation occurring in the outer rim acts to replace cell death occurring in the central necrotic core. This inward movement has been observed experimentally in vitro [11]. We note that in this case, mass is lost from the tumour due to the flow of cellular debris out of the tumour boundary. The dead material in the necrotic core undergoes exponential decay and acts as a mass source for the extracellular fluid phase (see Eqs. (2.7) and (2.8)). The flow of extracellular fluid is positive in the centre of the tumour, $x \in\left(0, R_{H}\right]$, but also flows into the tumour at the exterior boundary, providing the material required for cell proliferation at the outer rim of the tumour. The circulation pattern of the constituent phases at steady state is indicated by the arrows in Figure 2.

\subsection{Travelling wave solutions}

For large regions of parameter space, the model generates travelling wave solutions. That is, after an initial transient period during which the tumour composition is dynamic, the tumour profile settles to a steady 


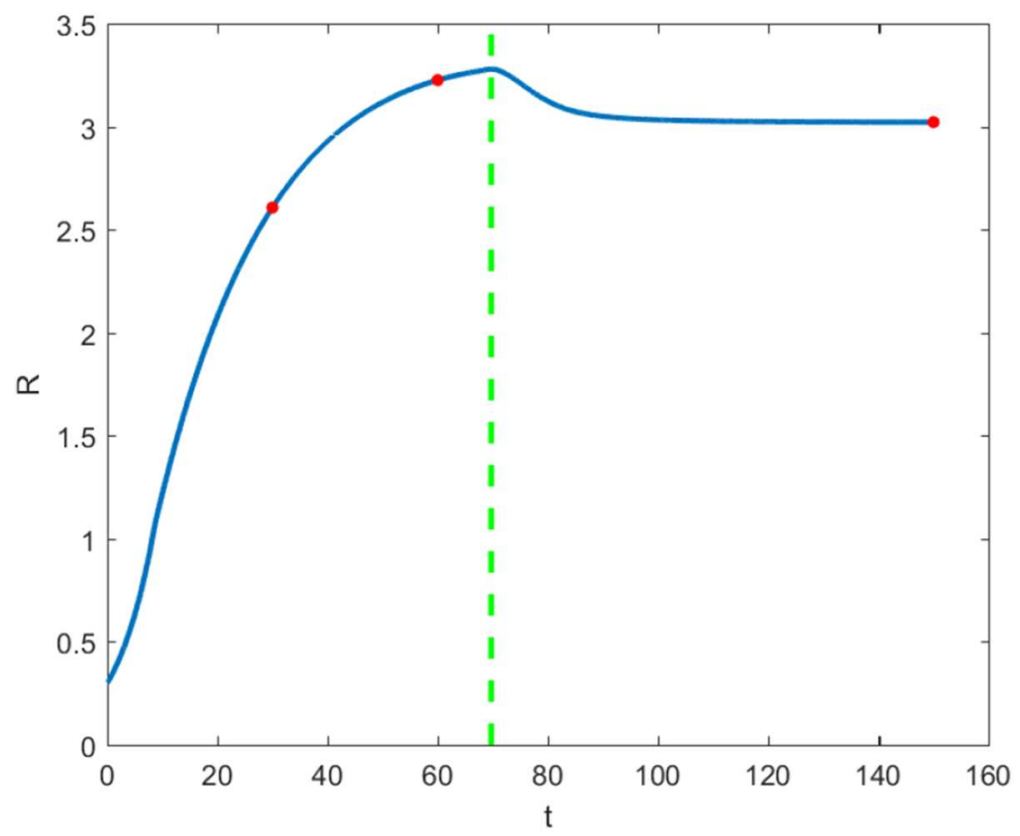

FIgURE 4. Tumour radius trajectory (blue line) for a simulation in which the steady state is reached after an initial 'overshoot', solving equations (2.22)-(2.32) and using the parameter values in Tables 3 and 4 , case iii. The red dots mark the time points $t=30,60,150$ for which we visualise snapshots of the internal tumour composition and phase velocities in Figure 5. The vertical dashed green line marks the time, $t_{\max }$, at which the tumour attains its maximum radius, $R_{\max }$.

composition which moves with a constant velocity as a travelling wave. This is evident from the trajectory of the tumour radius shown in Figure 3 for one such simulation exhibiting this behaviour.

Figure $3 \mathrm{~b}$ visualises a snapshot of the tumour compositions at 3 different time points throughout the simulation, as marked by the red points in Figure 3. At early times $(t=10)$ the tumour's spatial composition is similar to that depicted in Figure 2. As the tumour grows sufficiently large such that $c(0, t) \leq c_{N} \leq c_{H}$ we observe a large region of extensive necrosis at the centre of the tumour. As in Figure 2, this corresponds with a change of composition towards the centre of the tumour whereby, broadly speaking, $\frac{\partial \phi_{1}}{\partial x}>0$ and $\frac{\partial \phi_{2}}{\partial x}<0$ for $x<R_{H}$. However, in this case, as the tumour grows larger, the dead material phase at the centre of the tumour is degraded resulting in $\phi_{1}, \phi_{2} \ll 1$ at $x=0$ and the composition shown in Figure $3 \mathrm{~b}$ at $t=40$. This profile persists at later times and manifests as a travelling wave solution with constant wave speed. It is unclear which parameters/mechanisms determine the formation of a travelling wave as opposed to reaching a steady state tumour radius and this remains to be investigated in future work but we note that travelling waves have been reported for similar multiphase models [5, 22].

While it may be possible for spheroids to grow as travelling waves in vitro, we typically observe tumours both in vitro and in vivo that evolve to a steady state. As such, henceforth we focus on regions of parameter space that give rise to steady state solutions. We note also that travelling wave solutions of the type presented in Figure 3 are not indicative of an invasive tumour. For such an interpretation, the model would need to be adapted to consider a tumour growing into surrounding tissue, in contrast to the stress-free boundary conditions imposed here.

\section{3. 'Overshooting' the steady state}

Our three phase model also exhibits non-monotonic growth dynamics. The first non-monotonic behaviour that we characterise is shown by the trajectory in Figure 4. Here, the outer tumour radius initially increases 

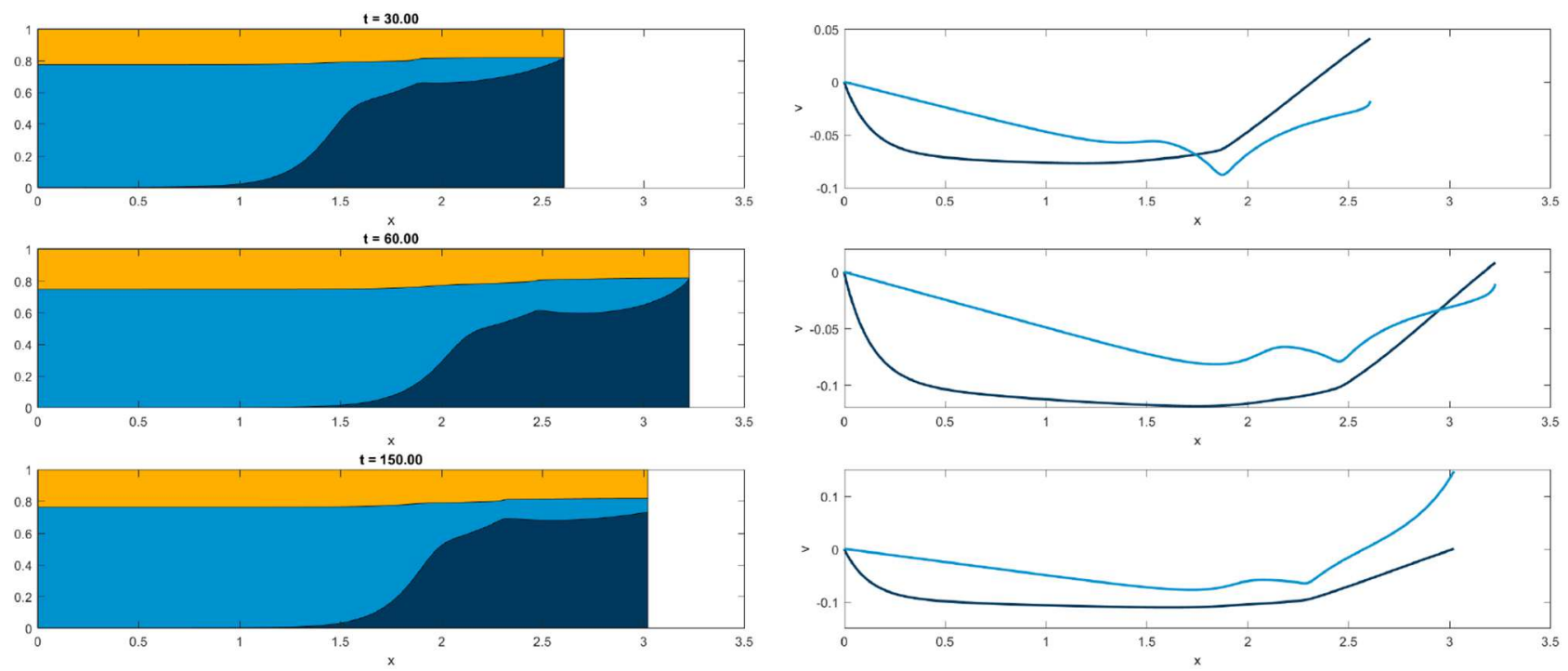

(A)

(B)

Figure 5. Time series showing the (a) internal tumour composition and (b) phase velocities $v_{1}$ and $v_{2}$ throughout the simulation resulting in 'overshoot' dynamics shown in Figure 4 . We observe a switch in the ordering of $v_{1}$ and $v_{2}$ at $x=R(t)$ between $t=60$ and $t=150$ which results in the non-monotonic trajectory. The volume fractions, $\phi_{i}$, and phase velocities, $v_{i}$, are represented as in Figure $2\left(v_{3}\right.$ not shown).

monotonically, overshooting its steady state size. The tumour then starts to regress and approaches its steady state radius monotonically from above. The dashed green line in Figure 4 marks the time $t_{\max }$ at which the tumour attains its maximum radius, $R_{\max }$. The red points indicate time points at which we visualise the corresponding tumour compositions in Figure $5 \mathrm{a}$ and phase velocities in Figure 5b.

The 'overshoot' in the outer tumour radius arises due to a change in the nature of the solution at the tumour boundary. During the initial growth phase, the tumour appears to approach steady state in a standard, monotonic manner with the phase velocity for the dead cells at the boundary less than the cell velocity there, so that $\frac{\mathrm{d} R}{\mathrm{~d} t}=v_{1}(R(t), t)>v_{2}(R(t), t)$ (see Fig. 5). However, as the velocity of the boundary decreases, it approaches the velocity of the dead material phase there. The turnaround point, at $t=t_{\max }$, occurs when $v_{1}(R(t), t)=v_{2}(R(t), t)$. For $t>t_{\max }, v_{1}(R(t), t)<v_{2}(R(t), t)$ so that there is an outflow of dead material on the tumour boundary. Thereafter dead material is lost from the interior of the tumour and the tumour decreases in volume. This continues until the tumour evolves to a steady state radius which is smaller than the maximum radius, $R_{\max }$. The steady state tumour composition is shown in Figure 5a and the tumour boundary is an outflow boundary for dead material at steady state. We note that the steady state tumour composition is similar to that presented in Section 3.1 (cf. Fig. 2) although the tumour's growth dynamics are very different.

\subsection{Damped oscillations about the steady state}

In Sections 3.1 and 3.3 we described tumour growth trajectories that ultimately approach a steady state solution monotonically, either from below or above. However, our model also admits solutions for which the tumour undergoes damped oscillations about the steady state radius. The long-term dynamics for one such simulation are shown in Figure 6.

In this case, the outer tumour boundary is an outflow boundary for the dead phase. In particular, $v_{2}(x, t)>0 \quad \forall x \in(0, R(t)]$, which leads to the gradual loss of this cellular debris from the interior of the tumour across the boundary. However this outward flow of non-viable cells results in a build up of dead material at the 
a)

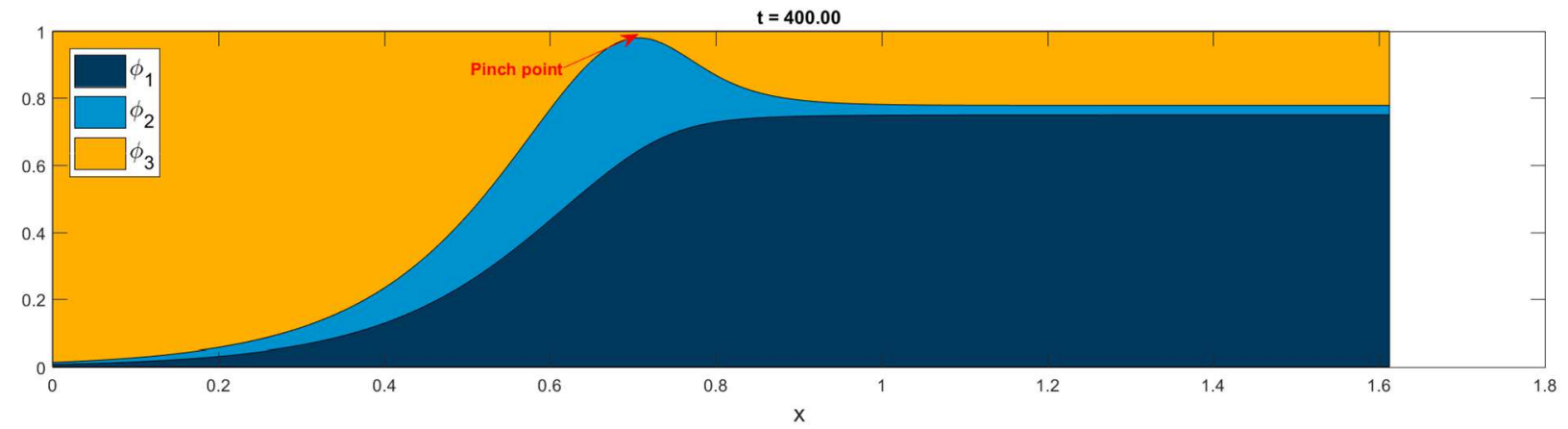

b)

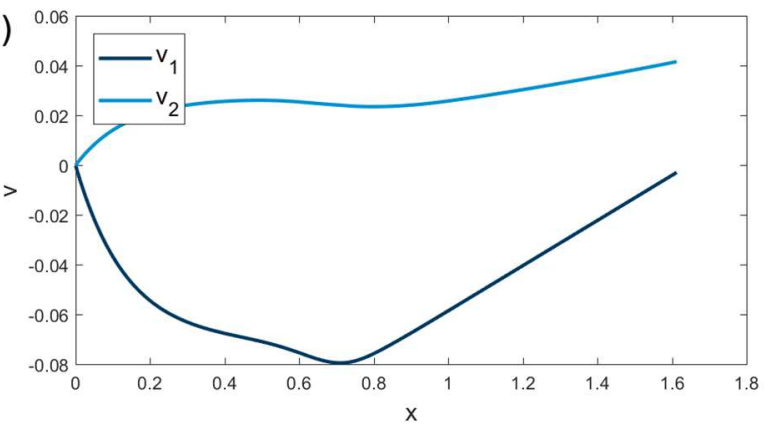

c)

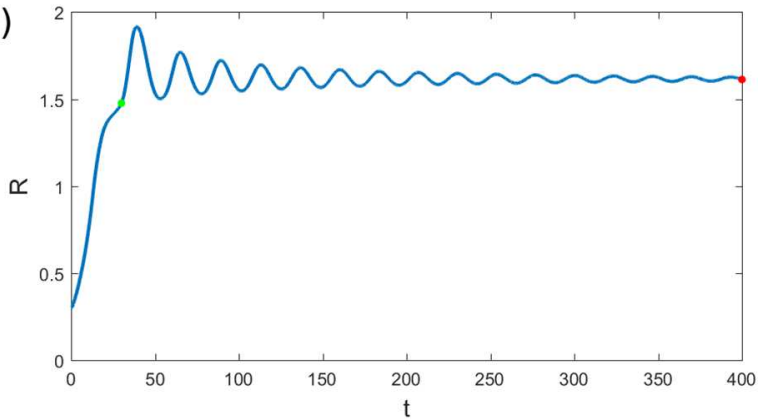

Figure 6. Example of a simulation of equations (2.22)-(2.32) for which the tumour undergoes damped oscillations about a steady state. (a) A snapshot of the internal tumour composition at $t=400$. The build up of the dead material phase at the edge of the necrotic core drives oscillatory dynamics and we annotate the 'pinch point' at which the fluid phase volume fraction $v_{3}$ is at its minimum. (b) The velocity profiles $v_{1}$ and $v_{2}$ at $t=400$. (c) The oscillatory trajectory of the outer tumour radius. The green and red dots mark the start and end points of the oscillations, respectively. The volume fractions, $\phi_{i}$, and phase velocities, $v_{i}$, are visualised as in Figure 2. The parameter values for this simulation are listed in Tables 3 and 4 , case iv.

edge of the necrotic core, where there is also a significant volume fraction of viable tumour cells, $\phi_{1}$. It is this internal build-up of dead material which drives the oscillations in tumour radius observed.

In our model, the material required for proliferation of the viable tumour cells is provided by the extracellular fluid phase and therefore the rate of proliferation is proportional to the volume fraction of this phase, $\phi_{3}=1-\phi_{1}-\phi_{2}$. In the previous simulations shown, at steady state a balance is reached within the tumour between the total new tumour mass from proliferation and the mass lost due to cell death. When the dead material builds up at the edge of the necrotic core, it creates a 'pinch point' at which $\phi_{3} \ll 1$, as annotated in Figure 6 . We introduce the notation $\epsilon(t)=\min _{x} \phi_{3}(x, t)$ and denote by $x_{\epsilon}(t)$ the position within the tumour at which this pinch point occurs. In Figure 7 we visualise the damped oscillations as a spiral in the $\left(x_{\epsilon}, \epsilon\right)$ phase plane. The start and end points of the oscillations are indicated by green and red points, respectively, in both Figures 6 and 7 .

The accumulation of dead material locally restricts tumour cell proliferation even where oxygen levels are high, and so the tumour volume decreases while the necrotic material clears. This corresponds to an increase in $\epsilon(t)$ and a decrease in $x_{\epsilon}$. Once sufficient necrotic material has been cleared the tumour cells then resume proliferation. The increased proliferation again results in the build up of dead material and the pinching off of the fluid phase, so that $\dot{\epsilon}<0$ (where ${ }^{\cdot}$ denotes the derivative with respect to $t$ ). Once the total proliferation 


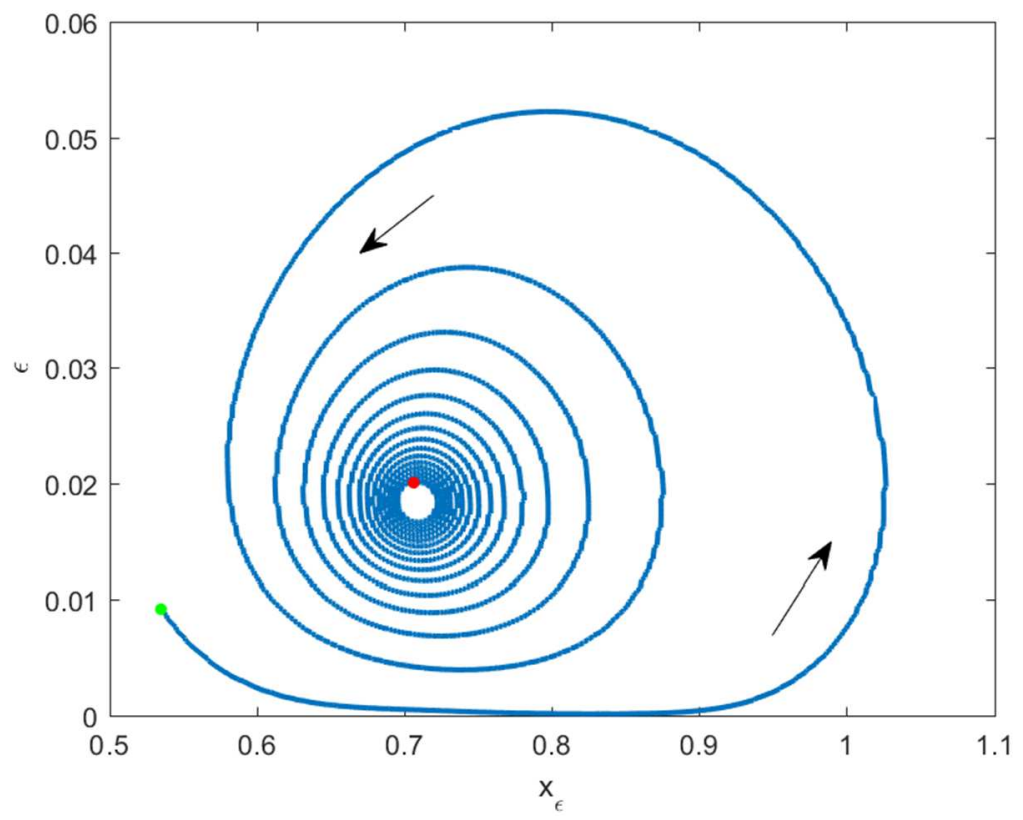

Figure 7. Phase plot in the $\left(x_{\epsilon}, \epsilon\right)$-plane of the oscillatory tumour dynamics, where $\epsilon(t)=$ $\min _{x} \phi_{3}(x, t)$ and $x_{\epsilon}(t)$ is the location of $\epsilon(t)$. The arrows show the direction of increasing $t$, and the green and red points mark the start and end of the trajectory, respectively.

within the tumour surpasses cell death, the tumour radius begins to increase again and correspondingly $\dot{x_{\epsilon}}>0$. This process drives the oscillatory dynamics observed in Figures 6 and 7.

\subsection{Parameter sweep results}

We catalogue all parameter combinations which generate steady state solutions and summarise the resulting equilibrium compositions in Figure 8. The right hand plot in Figure 8 shows the corresponding tumour radii at steady state for the aforementioned parameter sets. The blue bars give the position of the outer tumour radius at steady state, with the dashed green line representing $R_{0}$ for reference. Where applicable, we also display the positions of internal radii $R_{H}$ (red bars) and $R_{N}$ (yellow bars) as a summary of the steady state tumour composition. The radii $R_{H}$ and $R_{N}$ mark the spatial positions at which the oxygen concentration drops below the thresholds $c_{H}$ and $c_{N}$, respectively. We also mark the positions of key parameter sets $(\mathrm{A}, \mathrm{B}, \mathrm{C} \& \mathrm{D})$ which we investigate further in Section 4 (see Fig. 9).

The parameter combinations are sorted by the resulting steady state tumour radius. From visual inspection alone, it is difficult to determine any correlations between the parameter values and the tumour radius and composition at steady state, however we may make some general observations. The central columns of parameters, namely $\zeta, \phi^{*}$ and $\phi_{\min }$, relate to the cell-sensing activity in the tumour. Our simulations suggest that stronger cellular interactions (larger values of $\zeta$ ) combined with larger values of $\phi^{*}-\phi_{\min }$, the range of densities for which the cells experience adhesion, give rise to larger steady state solutions which do not become travelling wave solutions. Additionally, the more 'cell-like' the dead material phase is deemed to be, corresponding to large values of $\left(\theta_{\mu}, \theta_{p}, \theta_{\Sigma}\right)$, the more active material there is within the tumour, driving its evolution to a larger steady state radius. We observe this in the greater prominence of 'green' values in the bottom right of the heat map. We note also that low necrotic decay rates, $\lambda$, tend to give rise to smaller steady state tumours, whilst low rates of apoptosis, $\kappa$, and low drag coefficients with the fluid phase, $d_{i 3}$, tend to correspond to larger steady state tumours. 

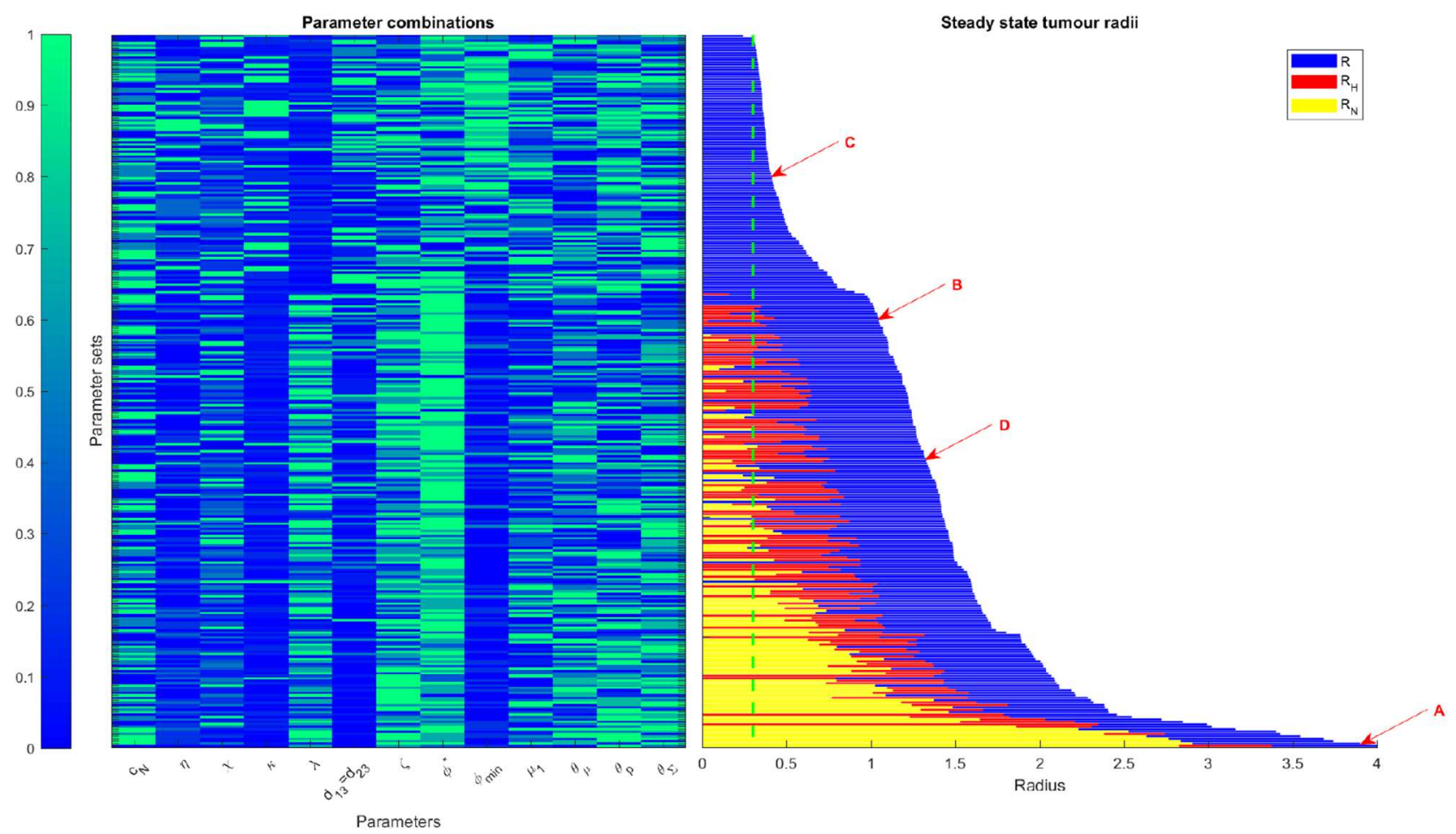

FIGURE 8. Catalogue of parameter combinations from the parameter sweep which generated steady state solutions. Left plot: heat map of parameter values with each row pertaining to a particular parameter set. The colour scale corresponds to the range of values included in the parameter sweep for each parameter (see Tab. 2), with green representing the maximum of the range and blue the minimum. Right plot: histogram of steady state tumour radii for the simulations corresponding to each row of the parameter heatmap. The blue bars show the steady state outer tumour radius, $R$, while the red and yellow bars show the positions of the internal radii $R_{H}$ and $R_{N}$, respectively. The dashed green line represents the initial condition $R_{0}$ for each simulation.

\section{The Influence OF THE DEAD MATERIAL ON TUMOUR GROWTH} DYNAMICS

As described in Section 2.2, the parameter vector $\boldsymbol{\theta}=\left(\theta_{\mu}, \theta_{p}, \theta_{\Sigma}\right)$ summarises the mechanical properties of the dead material phase relative to the tumour cell and extracellular fluid phases. Recall that the parameter $\theta_{\mu}$ specifies the relative viscosity of the dead phase (Eq. (2.10)), while the parameters $\theta_{p}$ and $\theta_{\Sigma}$ pertain to the cell-sensing properties of this phase (see Eq. (2.11)). In this section we perform a parameter sensitivity analysis in which the parameters $\theta_{\mu}, \theta_{p}$ and $\theta_{\Sigma}$ range across the unit cube. In this way we aim to determine how the manner in which we model the dead material phase affects the system dynamics. We note that the two corners of the cube, $(1,1,1)$ and $(0,0,0)$, correspond to two-phase limits of the full multiphase model.

We take a selection of parameter sets from the catalogue of values used to generate the steady state solutions described in Section 3. For each parameter combination, we perform a new parameter sweep over the sub-space $\boldsymbol{\theta} \in[0,1]^{3}$, holding all other parameters fixed. As such, we isolate, in each case, the dependence on the material properties of the dead phase of the growth dynamics given an original parameter combination for which the tumour evolves to a steady state. We visualise the resulting trajectories for tumour radius over time for a selection of 4 such parameter sweeps in Figure 9. The original parameter sets for each case are annotated in Figure 8 with the values listed in Table 4 , cases A-D. 
A)

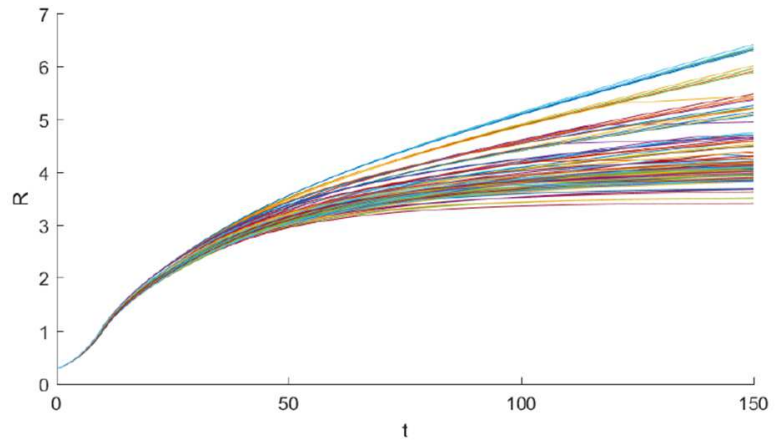

C)

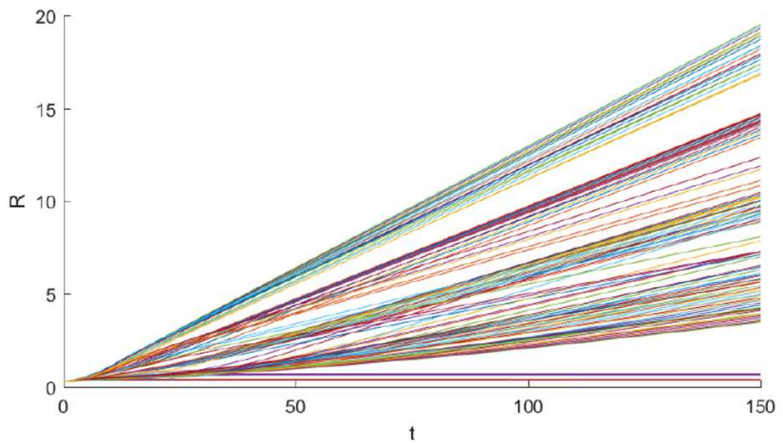

B)

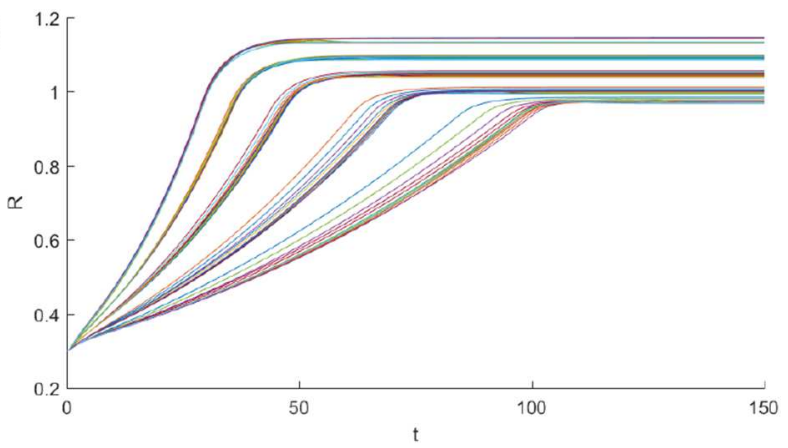

D)

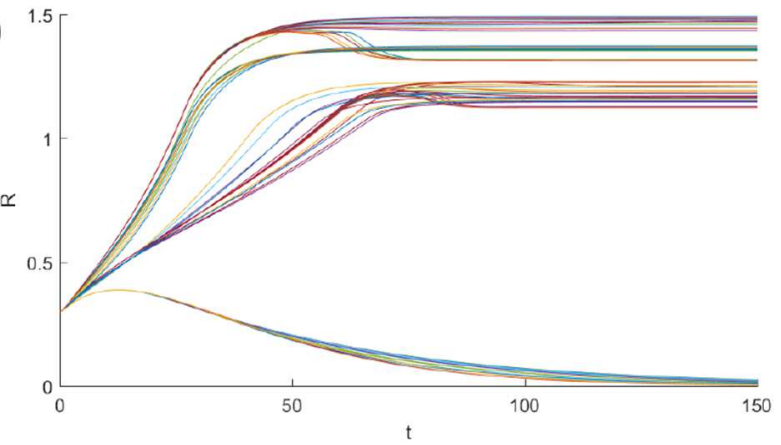

FIGURE 9. Tumour trajectories from a parameter sweep of the mechanical properties of the dead material phase, $\boldsymbol{\theta} \in[0,1]^{3}$ for 4 different cases, obtained by solving equations (2.22)-(2.32). All model parameters other than $\boldsymbol{\theta}=\left(\theta_{\mu}, \theta_{p}, \theta_{\Sigma}\right)$ remain fixed. The fixed parameter values for each case are listed in Table 4 , cases $\mathrm{A}-\mathrm{D}$, and have been correspondingly annotated in Figure 8.

The results presented in Figure 9 reveal that, for certain parameter sets, the qualitative growth dynamics can change markedly as we sweep across the sub-space $\boldsymbol{\theta} \in[0,1]^{3}$. In the rest of this section we analyse further the range of different effects observed.

\subsection{Qualitative dynamics insensitive to the properties of the dead material}

For certain parameter regimes, the properties of the dead material phase have little influence on the evolution of the tumour radius. In Figure 9A the growth dynamics are almost identical at early times. In this case, variation in $\boldsymbol{\theta}$ appears to modulate the rate at which the tumour approaches its steady state, with a few cases evolving to travelling wave solutions rather than reaching equilibrium.

We may summarise the proportion of the tumour volume at time $t$ that is comprised of each phase by defining the spatially averaged quantities

$$
\bar{\phi}_{i}(t)=\frac{1}{R(t)} \int_{0}^{R(t)} \phi_{i}(x, t) \mathrm{d} x .
$$

In Figure 10 we visualise the composition of the tumour at the end of the simulation for each parameter set. We sort the parameter combinations by final tumour radius and plot a heatmap of parameter sets as previously, with each row corresponding to a particular choice of $\boldsymbol{\theta}$. In the central plot of Figure 10 we visualise the final tumour composition, while the right-hand plot shows the final outer tumour radius, $R$, and the positions of the internal radii, $R_{H}$ and $R_{N}$. 

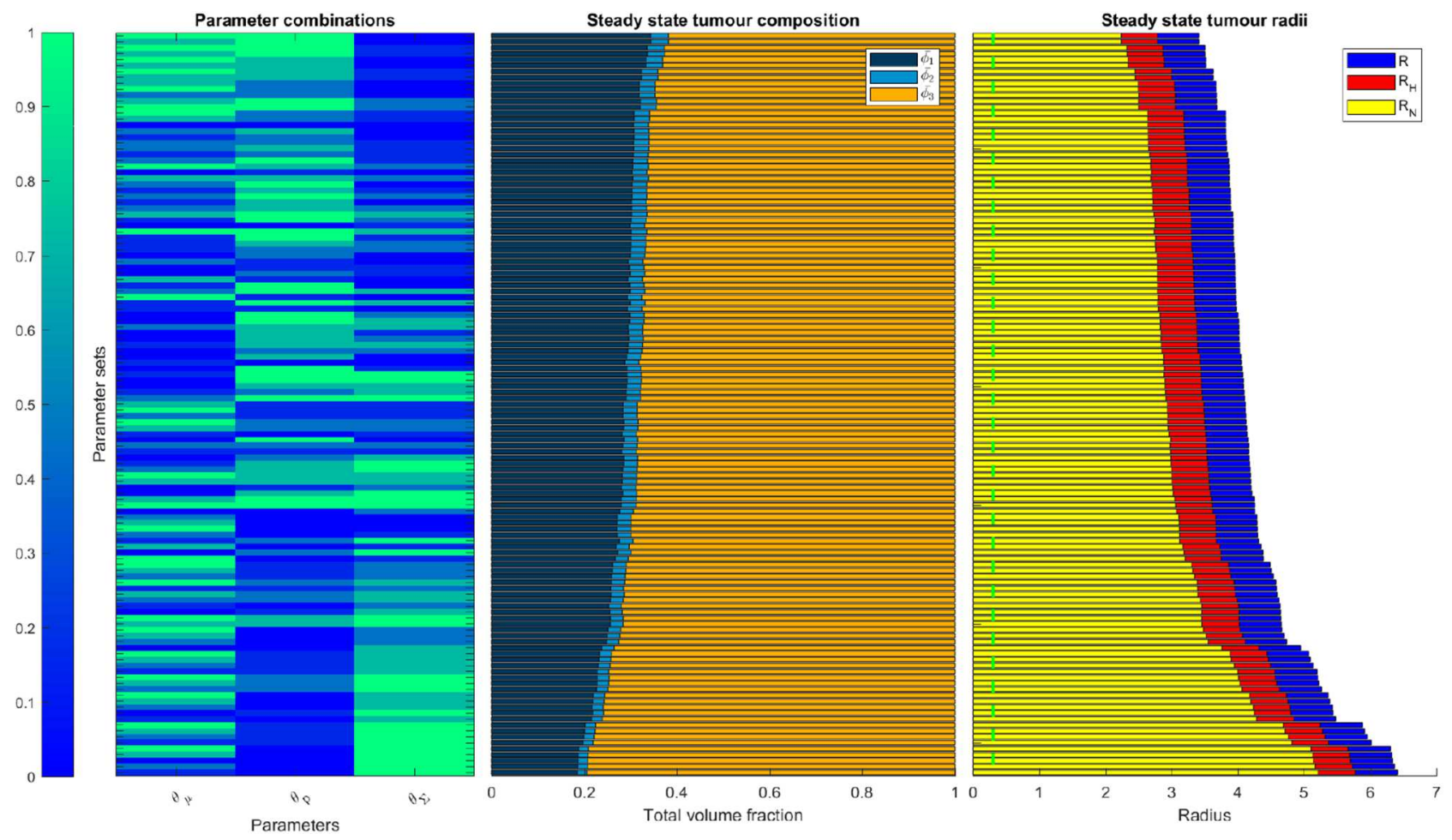

FIgURE 10. Summary of the steady state tumour compositions arising from case A of the $\boldsymbol{\theta}$-sweep (see Fig. 9). Left plot: heatmap of $\boldsymbol{\theta}$ parameter values corresponding to each simulation (visualisation as for Fig. 8). Central plot: proportion of the tumour volume at steady state comprised of each phase where $\bar{\phi}_{i}$ is defined by equation (4.1). The tumour cell, dead material and extracellular fluid phases are represented by the dark blue, light blue and yellow regions, respectively. Right plot: histogram showing the positions of the outer tumour radius, $R$ (blue), and the internal radii, $R_{H}$ (red) and $R_{N}$ (yellow), at steady state for each simulation. The vertical dashed green line marks the initial radius, $R_{0}$, for each simulation.

We notice that in this case, varying $\boldsymbol{\theta}$ does not significantly affect the resulting tumour composition. An increase in the steady state size of the outer tumour radius corresponds to a similar decrease in the average tumour cell volume fraction, $\bar{\phi}_{1}$. As such, all tumours have a similar total mass of tumour cells at steady state, and the parameter vector $\boldsymbol{\theta}$ acts to modulate the distribution of this material.

In Figure 9B, all trajectories exhibit the same qualitative behaviour and they group into 5 bands. This grouping suggests dominance of a single parameter or parameter combination. In Figure 11 we visualise the parameter combinations along with summary statistics for the steady state tumour composition, as previously. For this case the steady state tumour radii are not sufficiently large to create a necrotic core and so $R_{N}=0$ for all parameter combinations. The grouping of the tumours is also evident from the compositions at steady state. From the $\boldsymbol{\theta}$ parameter heatmap it is clear that the contribution of the dead phase to cell-sensing, $\theta_{\Sigma}$, is the dominant parameter, with larger values of $\theta_{\Sigma}$ yielding faster growing tumours which evolve to larger steady state sizes.

\subsection{Dead material has a quantitative effect on growth dynamics}

However there are cases for which variation in the $\boldsymbol{\theta}$ parameters has a more significant impact on the tumour growth dynamics. While the original parameter sets all gave rise to tumours which evolved to a 

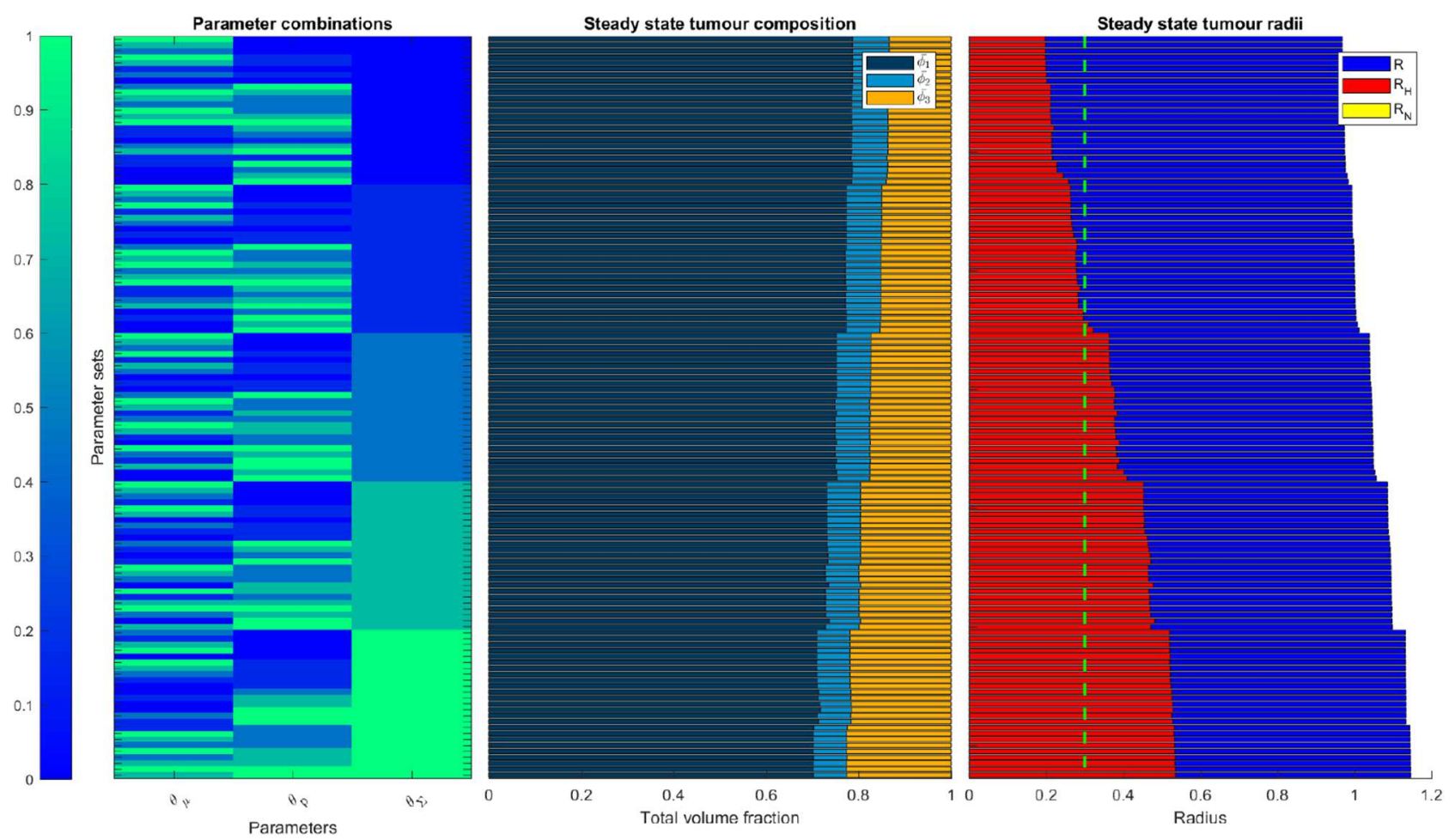

FiguRE 11. Summary of the steady state tumour compositions arising from case B of the $\boldsymbol{\theta}$-sweep (see Fig. 9). Visualisation for each plot as in Figure 10. NB: $R_{N}=0$ at steady state for all simulations in this case.

steady state, we see in case $\mathrm{C}$ in Figure 9 that most regions in the cube $\boldsymbol{\theta} \in[0,1]^{3}$ result in travelling wave solutions.

For this case, we may quantify the affect of the parameter vector $\boldsymbol{\theta}$ by considering the resulting wave speed. In Figure 12 we plot the velocity of the tumour boundary at the end of each simulation. We again notice that, for large values at least, $\theta_{\Sigma}$ is the dominant parameter in determining wave speed. Large values of $\theta_{\Sigma}$ create larger pressures in the tumour cell phase which thus drive faster tumour growth. When $\theta_{\Sigma}$ takes smaller values this effect is not so clear with the other two parameters, $\theta_{\mu}$ and $\theta_{p}$, having more of an influence on the dynamics. It is not clear why a small number of simulations reach a steady state rather than evolving to a travelling wave profile, with further investigation of this difference a direction for future work.

\subsection{Dead material has a qualitative effect on growth dynamics}

In Figure 9D, the mechanical properties of the dead material phase have a significant influence on the tumour growth dynamics. Monotonic and non-monotonic approaches to the steady state are observed for different values of $\left(\theta_{\mu}, \theta_{p}, \theta_{\Sigma}\right)$, while in other regions of parameter space the system is unable to sustain a viable tumour.

We illustrate the influence of the $\boldsymbol{\theta}$ parameters more clearly in Figure 13 by considering a sub-space of the unit cube. We traverse a line between two corners of the cube, taking $\theta_{\mu}=\theta_{p}=\theta_{\Sigma} \in[0,1]$. We note again that the corners $\boldsymbol{\theta}=(0,0,0)$ and $\boldsymbol{\theta}=(1,1,1)$ may be considered as two phase limits of our three phase model. We observe large variation in the qualitative tumour dynamics, with larger values of $\boldsymbol{\theta}$ corresponding to tumours 

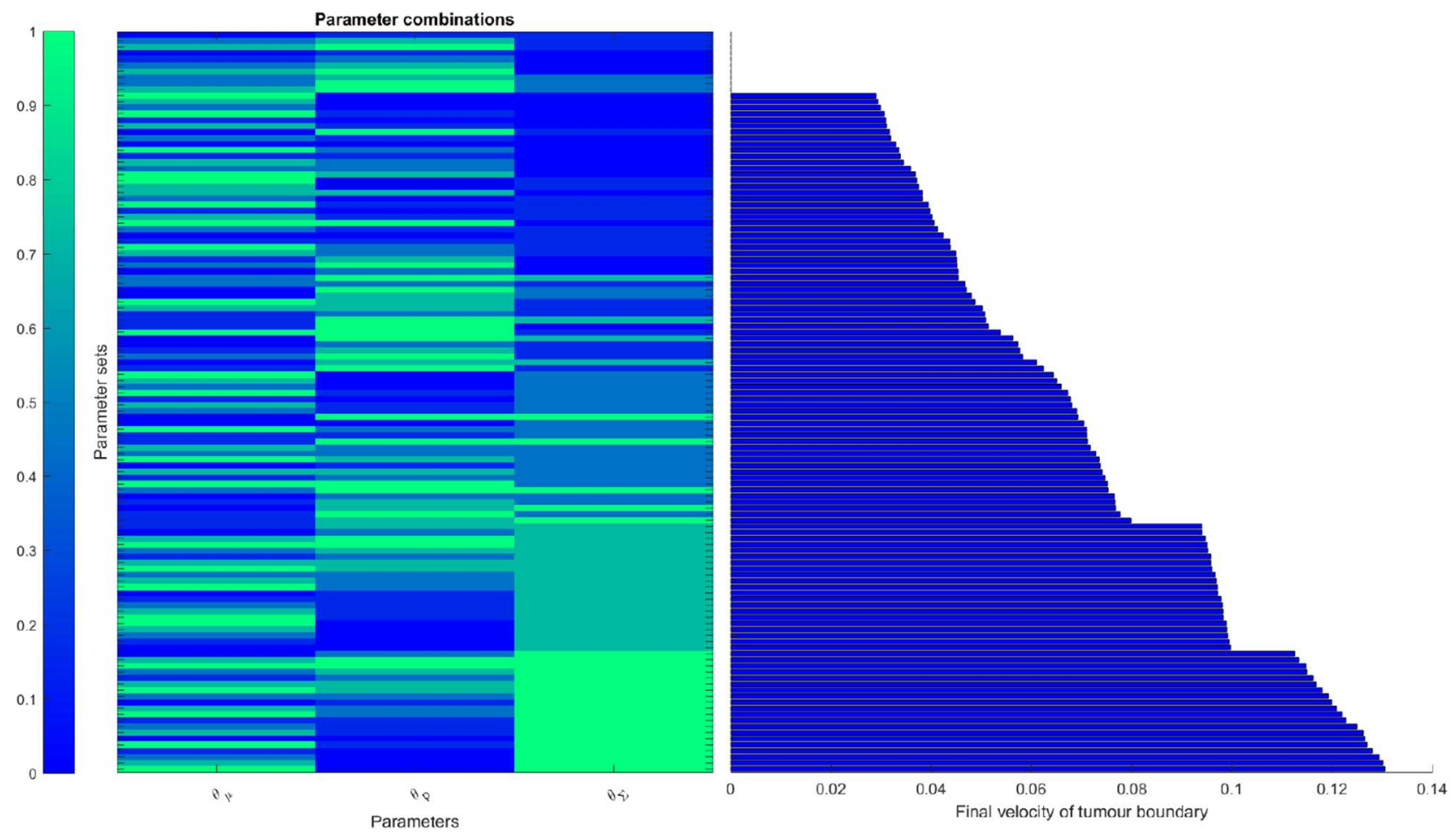

FiguRE 12. Visualisation of the travelling wave speeds arising for each simulation in case $\mathrm{C}$ of the $\boldsymbol{\theta}$ parameter sweep (see Fig. 9). Left plot: heatmap of $\boldsymbol{\theta}$ parameter values, visualised as in Figure 8. Right plot: histogram of the velocity of the tumour boundary at the end of each simulation.

that grow faster and evolve to larger equilibrium sizes. Conversely, smaller values of $\boldsymbol{\theta}$ yield smaller tumours, so that, in some cases, the system is unable to sustain a viable tumour ${ }^{2}$.

We conclude that the dead material and its mechanical properties can have a large influence on the qualitative growth dynamics. In particular, the limiting cases with $\theta_{\mu}=\theta_{p}=\theta_{\Sigma}=0$ and $\theta_{\mu}=\theta_{p}=\theta_{\Sigma}=1$ may give rise to markedly different growth trajectories.

\section{Discussion}

In this paper we have presented a new three phase model to investigate the influence of dead material on the growth dynamics and spatial structure of avascular tumours. We characterise the physical properties of the dead material as intermediate between those of the viable tumour cells and the extracellular fluid. The physical properties of this phase in relation to the tumour cell phase are summarised by 3 key model parameters, given by the vector $\boldsymbol{\theta}=\left(\theta_{\mu}, \theta_{p}, \theta_{\Sigma}\right)$.

Using numerical simulation of the model equations, we were able to reproduce biologically realistic tumour dynamics and spatial structures which are commonly observed in in vitro avascular tumour spheroids. In Section 3.1 we presented an example simulation result which showcased the heterogeneity in tumour composition and the complex internal dynamics which may occur in tumours at steady state. By contrast, in Section 3.2

\footnotetext{
${ }^{2}$ Simulations for small values of $\boldsymbol{\theta}$ resulted in numerical instability due to the volume fraction for the fluid phase, $\phi_{3}$, becoming very small upon contraction of the tumour radius. Accurately simulating this computationally would require a more sophisticated numerical scheme. Equally, the validity of the multiphase assumptions when one or more phases becomes extinct is also an open question to be addressed, but beyond the scope of the work presented in this paper.
} 


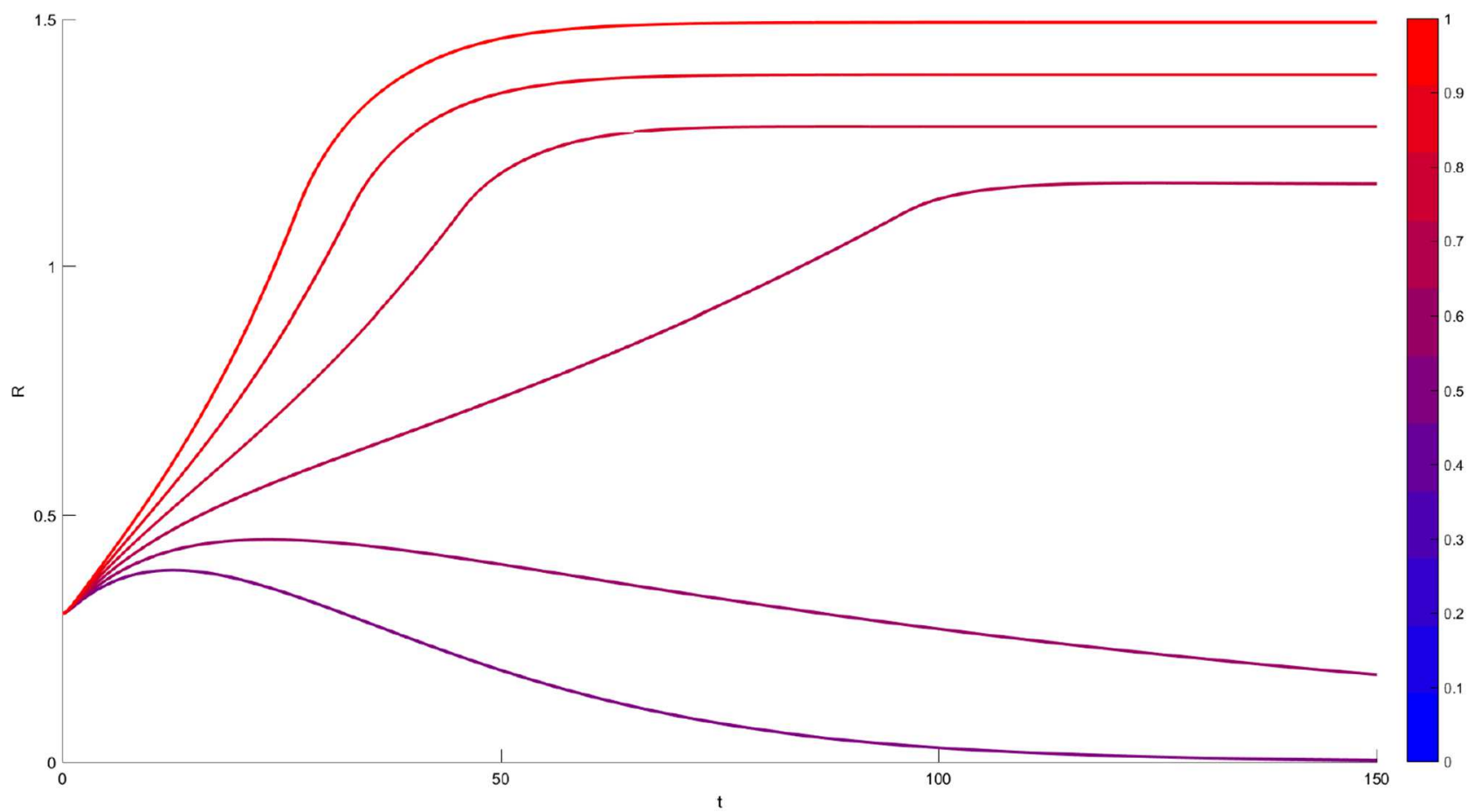

Figure 13. Tumour radius trajectories obtained by imposing $\theta_{\mu}=\theta_{p}=\theta_{\Sigma} \in[0,1]$ for case D in Figure 9. The colour scale corresponds to the value taken by each of the $\boldsymbol{\theta}$ parameters.

the results presented exhibited travelling wave dynamics. The complex interactions within the model which drives each of these contrasting qualitative dynamical behaviours is currently unclear. Further analysis of these mechanisms is a significant avenue for future work.

We further characterised some non-monotonic growth behaviours exhibited by the model which are driven by the intratumoural dynamics of the dead material phase. To the best of our knowledge, these dynamics have not been captured by existing models of avascular tumour growth. It is unclear whether some of these features may be artefacts of the 1D geometry used here. In particular, we may expect that the 'pinch-off' of the fluid phase resulting in oscillatory tumour dynamics may not be stable to symmetry-breaking perturbations in 2D and 3D. Future work on this model will include asymptotic analysis of these dynamics and extension of the numerics into 2D and/or 3D to further investigate the effects described here.

The local structure of the tumour vasculature is well-known to influence the growth of in vivo tumours $[4,9,17]$. In this model we have neglected explicit mention of the tumour vasculature and have focussed our attention on the impact of heterogeneity in internal tumour composition on the qualitative growth dynamics. Future work would include simulating the model in more complex geometries than the 1D cartesian simulations presented in this paper. In this setting the model could be further extended to incorporate the influence of tumour vasculature [20].

In Section 4 we investigated the influence of the dead phase on the tumour growth dynamics. For some cases considered, we observed large variations in tumour radius over time as we move around the parameter sub-space $\boldsymbol{\theta} \in[0,1]^{3}$. We conclude that the material properties of the non-viable cells, and the way in which we model them, may have a significant influence on the dynamics observed. This is of importance when using mathematical models as the basis for making predictions of tumour growth dynamics.

When therapies are administered to tumours they may induce further widespread cell death throughout the tumour, significantly altering the tumour composition. In light of the results presented here, we anticipate that 
the resulting redistribution of material between the tumour cell and dead material phases is likely to significantly impact the resulting regrowth dynamics and thus the observed response dynamics. In future work, we plan to extend the model presented here to incorporate therapeutic effects.

\section{Appendix A. Numerical scheme}

In this section we outline the numerical method that we used to solve equations (2.22)-(2.32). In Section A.1 we first map the system of equations to a fixed domain. In Sections A.2-A.5 we derive a finite difference scheme for each equation in turn. While there are many methods which could be employed to solve the given equations, we develop a simple, convergent finite difference scheme since we are interested in the qualitative behaviour of the model dynamics.

The numerical method is implemented on a fixed mesh in the fixed domain coordinates with $N$ equally-spaced nodes such that $\Delta \xi=\frac{1}{N-1}$. We index the nodes in space by $j=1, \ldots, N$ and denote $\xi_{j}=(j-1) \Delta \xi$. We solve the system of equations using explicit time-stepping with fixed timestep $\Delta t$ and denote the simulation timestep by $t_{n}=n \Delta t$. We introduce the following notation for variables evaluated at each mesh node and timestep, here using the volume fraction $\phi_{1}$ as an example:

$$
\phi_{1, j}^{n}=\phi_{1}\left(\xi_{j}, t_{n}\right) .
$$

\section{A.1 Fixed domain equations}

Equations (2.22)-(2.32) are defined on a growing domain with $0 \leq x \leq R(t)$. From a numerical standpoint, the growing domain makes it difficult to keep a mesh with equally-spaced nodes on which to solve the model equations. When solving these equations, it is convenient first to map them onto a fixed domain, $x \mapsto \xi \in[0,1]$, such that $\xi=\frac{x}{R(t)}$. We solve the transformed equations in these coordinates and then map back onto the growing domain when presenting simulation results. It is straightforward to show that the transformed equations can be written as follows:

$$
\begin{gathered}
\frac{\partial \phi_{1}}{\partial t}-\frac{\xi}{R} \frac{\mathrm{d} R}{\mathrm{~d} t} \frac{\partial \phi_{1}}{\partial \xi}+\frac{1}{R} \frac{\partial}{\partial \xi}\left(\phi_{1} v_{1}\right)=\eta \phi_{1}\left(1-\phi_{1}-\phi_{2}\right) H_{\epsilon}\left(c-c_{H}\right)-\chi \phi_{1} H_{\epsilon}\left(c_{N}-c\right)-\kappa \phi_{1}, \\
\frac{\partial \phi_{2}}{\partial t}-\frac{\xi}{R} \frac{\mathrm{d} R}{\mathrm{~d} t} \frac{\partial \phi_{2}}{\partial \xi}+\frac{1}{R} \frac{\partial}{\partial \xi}\left(\phi_{2} v_{2}\right)=\chi \phi_{1} H_{\epsilon}\left(c_{N}-c\right)+\kappa \phi_{1}-\lambda \phi_{2}, \\
0=\frac{D}{R^{2}} \frac{\partial^{2} c}{\partial \xi^{2}}-\Gamma \phi_{1} H_{\epsilon}\left(c-c_{H}\right), \\
0=\frac{4}{3} \frac{\mu_{1}}{R^{2}}\left(1-\phi_{1}\right) \frac{\partial}{\partial \xi}\left(\phi_{1} \frac{\partial v_{1}}{\partial \xi}\right)-\frac{4}{3} \frac{\theta_{\mu} \mu_{1}}{R^{2}} \phi_{1} \frac{\partial}{\partial \xi}\left(\phi_{2} \frac{\partial v_{2}}{\partial \xi}\right)+d_{12} \phi_{1} \phi_{2}\left(v_{2}-v_{1}\right) \\
-d_{13} \phi_{1}\left(\phi_{2} v_{2}+\left(1-\phi_{2}\right) v_{1}\right)+\frac{\theta_{p} \phi_{1} \phi_{2}}{R} \frac{\partial \Sigma_{\phi}}{\partial \xi}-\frac{\phi_{1}\left(1-\phi_{1}\right)}{R} \frac{\partial \Sigma_{\phi}}{\partial \xi}, \\
0=\frac{4}{3} \frac{\theta_{\mu} \mu_{1}}{R^{2}}\left(1-\phi_{2}\right) \frac{\partial}{\partial \xi}\left(\phi_{2} \frac{\partial v_{2}}{\partial \xi}\right)-\frac{4}{3} \frac{\mu_{1}}{R^{2}} \phi_{2} \frac{\partial}{\partial \xi}\left(\phi_{1} \frac{\partial v_{1}}{\partial \xi}\right)+d_{12} \phi_{1} \phi_{2}\left(v_{1}-v_{2}\right) \\
-d_{23} \phi_{2}\left(\phi_{1} v_{1}+\left(1-\phi_{1}\right) v_{2}\right)+\frac{\phi_{1} \phi_{2}}{R} \frac{\partial \Sigma_{\phi}}{\partial \xi}-\theta_{p} \frac{\phi_{2}\left(1-\phi_{2}\right)}{R} \frac{\partial \Sigma_{\phi}}{\partial \xi}, \\
\Sigma_{\phi}(\phi)=\frac{\zeta\left(\phi-\phi_{\min }\right)^{2}\left(\phi-\phi^{*}\right)}{(1-\phi)} H\left(\phi-\phi_{\min }\right),
\end{gathered}
$$




$$
\frac{\mathrm{d} R}{\mathrm{~d} t}=\left.v_{1}\right|_{\xi=1}
$$

with

$$
\begin{gathered}
\frac{\partial c}{\partial \xi}=0, \quad v_{1}=v_{2}=0 \quad \text { at } \quad \xi=0 \\
\phi_{2}^{+}=0, \quad c=c_{\infty} \quad \text { at } \quad \xi=1 \\
\frac{4}{3} \frac{\mu_{1}}{R} \frac{\partial v_{1}}{\partial \xi}-\Sigma_{\phi}\left(\phi_{1}+\theta_{\Sigma} \phi_{2}\right)=\frac{4}{3} \frac{\theta_{\mu} \mu_{1}}{R} \frac{\partial v_{2}}{\partial \xi}-\theta_{p} \Sigma_{\phi}\left(\phi_{1}+\theta_{\Sigma} \phi_{2}\right)=0 \quad \text { at } \quad \xi=1, \\
\phi_{1}=\tilde{\phi}_{1}(\xi), \quad \phi_{2}=\tilde{\phi}_{2}(\xi), \quad R=R_{0} \quad \text { at } \quad t=0 .
\end{gathered}
$$

and

The numerical methods for each equation are combined to solve the full system of equations via the pseudocode presented in Algorithm 1.

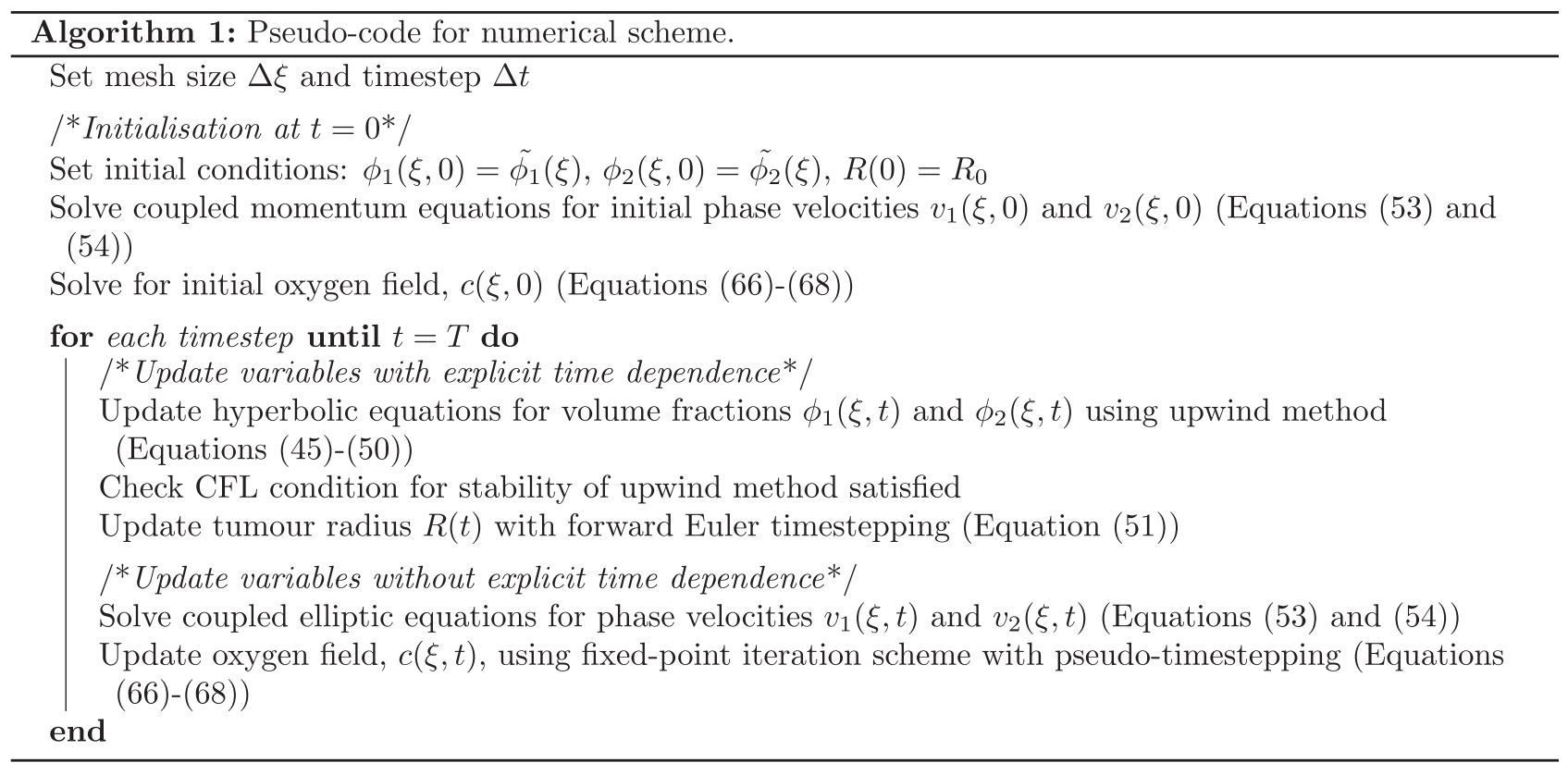

\section{A.2 Upwinding for conservation of mass equations (Eqs. (A.1) and (A.2))}

\section{A.2.1 Interior nodes}

We solve these hyperbolic equations using upwinding for the advection terms and standard, central differences for the non-advection terms [24]. Since the phase velocities, $v_{i}$, are not constant in $\xi$, the direction of advection is given by the sign of $v_{i}-\xi \frac{\mathrm{d} R}{\mathrm{~d} t}$ at each node. As such we take care to choose the correct upwinding formulation in each case. Note that since the tumour boundary moves with the tumour phase velocity, $\frac{\mathrm{d} R^{n}}{\mathrm{~d} t}=v_{1, N}^{n}$. The finite difference scheme for the interior nodes, $j=2, \ldots, N-1$, is given below. 
Case: $\frac{1}{R^{n}}\left(v_{i, j}^{n}-\xi_{j}^{n} v_{1, N}^{n}\right)>0$

$$
\phi_{i, j}^{n+1}=\phi_{i, j}^{n}-\frac{1}{R^{n}}\left(v_{i, j}^{n}-\xi_{j}^{n} v_{1, N}^{n}\right) \frac{\Delta t}{\Delta \xi}\left(\phi_{i, j}^{n}-\phi_{i, j-1}^{n}\right)-\frac{\Delta t}{R^{n}} \phi_{i, j}^{n} \frac{1}{2 \Delta \xi}\left(v_{i, j+1}^{n}-v_{i, j-1}^{n}\right)+\Delta t S_{i, j}^{n}
$$

Case: $\frac{1}{R^{n}}\left(v_{i, j}^{n}-\xi_{j}^{n} v_{1, N}^{n}\right)<0$

$$
\phi_{i, j}^{n+1}=\phi_{i, j}^{n}-\frac{1}{R^{n}}\left(v_{i, j}^{n}-\xi_{j}^{n} v_{1, N}^{n}\right) \frac{\Delta t}{\Delta \xi}\left(\phi_{i, j+1}^{n}-\phi_{i, j}^{n}\right)-\frac{\Delta t}{R^{n}} \phi_{i, j}^{n} \frac{1}{2 \Delta \xi}\left(v_{i, j+1}^{n}-v_{i, j-1}^{n}\right)+\Delta t S_{i, j}^{n} .
$$

Since we are using an upwinding scheme, we must check at each timestep that equations (A.12) and (A.13) satisfy the CFL stability condition [24]. In this case we require that $\left|\frac{1}{R}\left(v_{i}-\xi \frac{\mathrm{d} R}{\mathrm{~d} t}\right)\right| \frac{\Delta t}{\Delta \xi} \leq 1$ for all nodes.

\section{A.2.2 Boundary nodes}

We exploit the fact that $\xi=0$ is a characteristic and use forward differences to solve the characteristic equation for $\phi_{i, 1}$. We note also that symmetry about the origin gives $v_{i, 1}=0$. Thus our finite difference scheme at $\xi_{1}=0$ is

$$
\phi_{i, 1}^{n+1}=\phi_{i, 1}^{n}+\Delta t\left(-\frac{\phi_{i, 1}^{n}}{R^{n}} \frac{v_{i, 2}^{n}}{\Delta \xi}+S_{i, 1}^{n}\right)
$$

At the exterior boundary, $\xi=1$, for $\phi_{1}$ we use the fact that it is also characteristic (since the boundary moves with the phase velocity) and use a backward difference for $v_{1, N}$. The corresponding difference equation is given by

$$
\phi_{1, N}^{n+1}=\phi_{1, N}^{n}+\Delta t\left(-\frac{\phi_{1, N}^{n}}{R^{n}} \frac{\left(v_{1, N}^{n}-v_{1, N-1}^{n}\right)}{\Delta \xi}+S_{1, N}^{n}\right)
$$

At $\xi=1$ for $\phi_{2}$ there are two cases to consider since the exterior boundary is no longer a characteristic. For an outflow boundary, where $v_{2, N}^{n}>v_{1, N}^{n}$, we use the usual upwind equation with a one-sided backward difference for $\phi_{2}$ :

$$
\phi_{2, N}^{n+1}=\phi_{2, N}^{n}-\frac{1}{R} \frac{\Delta t}{\Delta \xi}\left(v_{2, N}^{n}-v_{1, N}^{n}\right)\left(\phi_{2, N}^{n}-\phi_{2, N-1}^{n}\right)+\Delta t\left(-\frac{\phi_{2, N}^{n}}{R^{n}} \frac{\left(v_{2, N}^{n}-v_{2, N-1}^{n}\right)}{\Delta \xi}+S_{2, N}^{n}\right) .
$$

For an inflow boundary, where $v_{2, N}^{n}<v_{1, N}^{n}$, we also use upwinding but impose the additional boundary condition $\phi_{2}^{+}=0$ as described in the main text. Thus, our 'ghost node' at " $j=N+1$ " is 0 and we have

$$
\phi_{2, N}^{n+1}=\phi_{2, N}^{n}-\frac{1}{R} \frac{\Delta t}{\Delta \xi}\left(v_{2, N}^{n}-v_{1, N}^{n}\right)\left(-\phi_{2, N}^{n}\right)+\Delta t\left(-\frac{\phi_{2, N}^{n}}{R^{n}} \frac{\left(v_{2, N}^{n}-v_{2, N-1}^{n}\right)}{\Delta \xi}+S_{2, N}^{n}\right) .
$$

\section{A.3 Forward difference for $\mathbf{R}(\mathrm{t})$ (Eq. (A.7))}

We use an explicit scheme to solve Equation (A.7) so that

$$
R^{n+1}=R^{n}+\Delta t v_{1, N}^{n} .
$$




\section{A.4 Coupled equations for conservation of momentum (Eqs. (A.4) and (A.5))}

\section{A.4.1 Interior nodes}

We discretise $\frac{\partial}{\partial \xi}\left(\phi_{i} \frac{\partial v_{i}}{\partial \xi}\right)$ using a central difference at half grid points in order to obtain a symmetric approximation [24]. We use a Taylor expansion and one-sided differences to approximate $\phi_{i, j \pm \frac{1}{2}}$. In this way we obtain the discretisation in equation (A.19) which we denote by $\Delta_{i, j}$ for convenience, where $i$ indexes the phase and $j$ the central node of the approximation. For derivatives of $\Sigma_{\phi}$ we use standard central differences.

$$
\begin{aligned}
\frac{\partial}{\partial \xi}\left(\phi_{i} \frac{\partial v_{i}}{\partial \xi}\right) \approx & \frac{1}{\Delta \xi^{2}}\left[\frac{1}{2}\left(\phi_{i, j}^{n+1}+\phi_{i, j-1}^{n+1}\right) v_{i, j-1}^{n+1}-\left(\frac{1}{2} \phi_{i, j-1}^{n+1}+\phi_{i, j}^{n+1}+\frac{1}{2} \phi_{i, j+1}^{n+1}\right) v_{i, j}^{n+1}\right. \\
& \left.+\frac{1}{2}\left(\phi_{i, j}^{n+1}+\phi_{i, j+1}^{n+1}\right) v_{i, j+1}^{n+1}\right]=: \Delta_{i, j} .
\end{aligned}
$$

Applying these approximations to equations (A.4) and (A.5) yields the following finite difference equations:

$$
\begin{aligned}
0= & -\phi_{1, j}^{n+1} \frac{\left(1-\phi_{1, j}^{n+1}\right)}{R^{n+1}} \frac{1}{2 \Delta \xi}\left(\sum_{\phi, j+1}^{n+1}-\Sigma_{\phi, j-1}^{n+1}\right)+\frac{4}{3}\left(1-\phi_{1, j}^{n+1}\right) \frac{\mu_{1}}{\left(R^{n+1}\right)^{2}} \Delta_{1, j} \\
& +\frac{\theta_{p} \phi_{1, j}^{n+1} \phi_{2, j}^{n+1}}{\left(R^{n+1}\right)^{2}} \frac{1}{2 \Delta \xi}\left(\sum_{\phi, j+1}^{n+1}-\Sigma_{\phi, j-1}^{n+1}\right)-\frac{4}{3} \frac{\theta_{\mu} \mu_{1}}{\left(R^{n+1}\right)^{2}} \phi_{1, j}^{n+1} \Delta_{2, j} \\
& +d_{12} \phi_{1, j}^{n+1} \phi_{2, j}^{n+1}\left(v_{2, j}^{n+1}-v_{1, j}^{n+1}\right)-d_{13} \phi_{1, j}^{n+1}\left(\phi_{2, j}^{n+1} v_{2, j}^{n+1}+\left(1-\phi_{2, j}^{n+1}\right) v_{1, j}^{n+1}\right), \\
0= & -\theta_{p} \phi_{2, j}^{n+1} \frac{\left(1-\phi_{2, j}^{n+1}\right)}{R^{n+1}} \frac{1}{2 \Delta \xi}\left(\Sigma_{\phi, j+1}^{n+1}-\Sigma_{\phi, j-1}^{n+1}\right)+\frac{4}{3}\left(1-\phi_{2, j}^{n+1}\right) \frac{\theta_{\mu} \mu_{1}}{\left(R^{n+1}\right)^{2}} \Delta_{2, j} \\
& +\frac{\phi_{1, j}^{n+1} \phi_{2, j}^{n+1}}{\left(R^{n+1}\right)^{2}} \frac{1}{2 \Delta \xi}\left(\Sigma_{\phi, j}^{n+1}-\Sigma_{\phi, j-1}^{n+1}\right)-\frac{4}{3} \frac{\mu_{1}}{\left(R^{n+1}\right)^{2}} \phi_{2, j}^{n+1} \Delta_{1, j} \\
& +d_{12} \phi_{1, j}^{n+1} \phi_{2, j}^{n+1}\left(v_{1, j}^{n+1}-v_{2, j}^{n+1}\right)-d_{23} \phi_{2, j}^{n+1}\left(\phi_{1, j}^{n+1} v_{1, j}^{n+1}+\left(1-\phi_{1, j}^{n+1}\right) v_{2, j}^{n+1}\right) .
\end{aligned}
$$

We note that in equations (A.20) and (A.21), $\Sigma_{\phi, j}$ is evaluated at $\left(\phi_{1, j}+\theta_{\Sigma} \phi_{2, j}\right)$.

Equations (A.20) and (A.21) comprise a coupled linear system which may alternatively be written in the form $M \boldsymbol{v}=\boldsymbol{d}$ for the velocity vector $\boldsymbol{v}=\left[\boldsymbol{v}_{\mathbf{1}}, \boldsymbol{v}_{\mathbf{2}}\right]^{T}$. The matrix, M, is of 'block tridiagonal' form such that

$$
M=\left(\begin{array}{l|l}
A & B \\
\hline C & D
\end{array}\right)
$$

where $A, B, C, D$ are tridiagonal matrices defined by:

$$
\begin{aligned}
& A_{j, j}=-\frac{4}{3}\left(1-\phi_{1, j}^{n+1}\right) \frac{\mu_{1}}{\left(R^{n+1}\right)^{2}} \frac{1}{\Delta \xi^{2}}\left(\frac{1}{2} \phi_{1, j-1}^{n+1}+\phi_{1, j}^{n+1}+\frac{1}{2} \phi_{1, j+1}^{n+1}\right)-\phi_{1, j}^{n+1}\left(d_{12} \phi_{2, j}^{n+1}+d_{13}\left(1-\phi_{2, j}^{n+1}\right)\right), \\
& A_{j, j \pm 1}=\frac{4}{3}\left(1-\phi_{1, j}^{n+1}\right) \frac{\mu_{1}}{\left(R^{n+1}\right)^{2}} \frac{1}{2 \Delta \xi^{2}}\left(\phi_{1, j}^{n+1}+\phi_{1, j \pm 1}^{n+1}\right), \\
& B_{j, j}=\frac{4}{3} \frac{\theta_{\mu} \mu_{1}}{\left(R^{n+1}\right)^{2}} \phi_{1, j}^{n+1} \frac{1}{\Delta \xi^{2}}\left(\frac{1}{2} \phi_{2, j-1}^{n+1}+\phi_{2, j}^{n+1}+\frac{1}{2} \phi_{2, j+1}^{n+1}\right)+\left(d_{12}-d_{13}\right) \phi_{1, j}^{n+1} \phi_{2, j}^{n+1}, \\
& B_{j, j \pm 1}=\frac{4}{3} \frac{\theta_{\mu} \mu_{1}}{\left(R^{n+1}\right)^{2}} \phi_{1, j}^{n+1} \frac{1}{2 \Delta \xi^{2}}\left(\phi_{2, j}^{n+1}+\phi_{2, j \pm 1}^{n+1}\right), \\
& C_{j, j}=\frac{4}{3} \frac{\mu_{1}}{\left(R^{n+1}\right)^{2}} \phi_{2, j}^{n+1} \frac{1}{\Delta \xi^{2}}\left(\frac{1}{2} \phi_{1, j-1}^{n+1}+\phi_{1, j}^{n+1}+\frac{1}{2} \phi_{1, j+1}^{n+1}\right)+\left(d_{12}-d_{23}\right) \phi_{1, j}^{n+1} \phi_{2, j}^{n+1},
\end{aligned}
$$




$$
\begin{aligned}
& C_{j, j \pm 1}=\frac{4}{3} \frac{\mu_{1}}{\left(R^{n+1}\right)^{2}} \phi_{2, j}^{n+1} \frac{1}{2 \Delta \xi^{2}}\left(\phi_{1, j}^{n+1}+\phi_{1, j \pm 1}^{n+1}\right), \\
& D_{j, j}=-\frac{4}{3}\left(1-\phi_{2, j}^{n+1}\right) \frac{\theta_{\mu} \mu_{1}}{\left(R^{n+1}\right)^{2}} \frac{1}{\Delta \xi^{2}}\left(\frac{1}{2} \phi_{2, j-1}^{n+1}+\phi_{2, j}^{n+1}+\frac{1}{2} \phi_{2, j+1}^{n+1}\right)-\phi_{2, j}^{n+1}\left(d_{12} \phi_{1, j}^{n+1}+d_{23}\left(1-\phi_{1, j}^{n+1}\right)\right), \\
& D_{j, j \pm 1}=\frac{4}{3}\left(1-\phi_{2, j}^{n+1}\right) \frac{\theta_{\Sigma} \mu \mu_{1}}{\left(R^{n+1}\right)^{2}} \frac{1}{2 \Delta \xi^{2}}\left(\phi_{2, j}^{n+1}+\phi_{2, j \pm 1}^{n+1}\right) .
\end{aligned}
$$

\section{A.4.2 Boundary nodes}

We combine the discretisation for the interior nodes with the boundary conditions at the boundaries $\xi=0$ and $\xi=1$. The symmetry conditions at $\xi=0$ give:

$$
v_{\alpha, 1}^{n+1}=v_{\beta, 1}^{n+1}=0 .
$$

We apply backward differences for the no-stress conditions at the exterior boundary $\xi=1$ :

$$
\begin{aligned}
& v_{1, N}^{n+1}=v_{1, N-1}^{n+1}+\frac{3}{4} \frac{R^{n+1}}{\mu_{1}} \Delta \xi \Sigma_{\phi, N}^{n+1}, \\
& v_{2, N}^{n+1}=v_{2, N-1}^{n+1}+\frac{3}{4} \frac{\theta_{p} R^{n+1}}{\theta_{\mu} \mu_{1}} \Delta \xi \Sigma_{\phi, N}^{n+1} .
\end{aligned}
$$

\section{A.5 Fixed-point iteration scheme for c with pseudo-timestepping (Eq. (A.3))}

\section{A.5.1 Interior nodes}

We use a fixed-point iteration scheme to deal with the non-linearity in equation (A.3) arising from the tanh term. That is, for a non-linear system $A(\boldsymbol{u}) \boldsymbol{u}=b(\boldsymbol{u})$ we perform iterations, indexed by $k$, solving the linear system $A\left(\boldsymbol{u}^{k}\right) \boldsymbol{u}^{k+1}=b\left(\boldsymbol{u}^{k}\right)$ until convergence is reached. We combine this fixed-point iteration scheme with a 'pseudo-timestep', $\rho$, in order to iterate towards the quasi-steady state solution. We use an implicit scheme for this pseudo-timestepping and so we solve the following finite difference scheme for $c^{k+1}$ :

$$
\frac{1}{\rho}\left(c_{j}^{k+1}-c_{j}^{k}\right)=\frac{D}{\left(R^{n+1}\right)^{2}} \frac{1}{(\Delta \xi)^{2}}\left(c_{j+1}^{k+1}-2 c_{j}^{k+1}+c_{j-1}^{k+1}\right)-\frac{\Gamma}{2} \phi_{1, j}^{n+1}\left(1+\tanh \left(\frac{c_{j}^{k}-c_{N}}{\epsilon}\right)\right) .
$$

We assume convergence to have occurred at iteration $k$ when $\max _{j}\left|c_{j}^{k}-c_{j}^{k-1}\right|$ falls below a pre-determined threshold, at which point we define $c^{n+1}=c^{k}$.

\section{A.5.2 Boundary nodes}

The linearised system in equation (A.33) is solved with the boundary conditions at $\xi=0$ and $\xi=1$ at each iteration. The symmetry boundary condition at $\xi=0$ gives:

$$
c_{1}^{k+1}=c_{2}^{k+1},
$$

while the Dirichlet condition at the exterior boundary, $\xi=1$, gives:

$$
c_{N}^{k+1}=c_{\infty} .
$$


Acknowledgements. This work was supported by the Engineering and Physical Sciences Research Council [grant number EP/G037280/1]. TL would also like to thank the Moffitt Cancer Center, where some of this work was undertaken, for their hospitality.

\section{REFERENCES}

[1] D. Ambrosi and L. Preziosi, Cell adhesion mechanisms and stress relaxation in the mechanics of tumours. Biomech. Model. Mechanobiol. 8 (2009) 397-413.

[2] R.P. Araujo and D.L.S. McElwain, A mixture theory for the genesis of residual stresses in growing tissues I: a general formulation. SIAM J. Appl. Math. 65 (2005) 1261-1284.

[3] R.P. Araujo and D.L.S. McElwain, A mixture theory for the genesis of residual stresses in growing tissues II: solutions to the biphasic equations for a multicell spheroid. SIAM J. Appl. Math. 66 (2005) 447-467.

[4] J.A. Bertout, S.A. Patel and M.C. Simon, The impact of $\mathrm{O}_{2}$ availability on human cancer. Nat. Rev. Cancer 8 (2008) $967-975$.

[5] C.J. Breward, H.M. Byrne and C.E. Lewis, The role of cell-cell interactions in a two-phase model for a vascular tumour growth. J. Math. Biol. 45 (2002) 125-152.

[6] J.M. Brown and A.J. Giaccia, The unique physiology of solid tumors: opportunities (and problems) for cancer therapy. Cancer Res. 58 (1998) 1408-1416.

[7] H. Byrne and M. Chaplain, Necrosis and apoptosis: distinct cell loss mechanisms in a mathematical model of avascular tumour growth. J. Theor. Med. 1 (1998) 223-235.

[8] H.M. Byrne, J.R. King, D.L.S. McElwain and L. Preziosi, A two-phase model of solid tumour growth. Appl. Math. Lett. 16 (2003) 567-573.

[9] P. Carmeliet and R.K. Jain, Angiogenesis in cancer and other diseases. Nature 407 (2000) 249-257.

[10] E.J. Crampin, E.A. Gaffney and P.K. Maini, Reaction and diffusion on growing domains: scenarios for robust pattern formation. Bull. Math. Biol. 61 (1999) 1093-1120.

[11] M.J. Dorie, R.F. Kallman, D.F. Rapacchietta, D. Van Antwerp and Y.R. Huang, Migration and internalization of cells and polystyrene microspheres in tumor cell spheroids. Exp. Cell Res. 141 (1982) 201-209.

[12] D. Eriksson and T. Stigbrand, Radiation-induced cell death mechanisms. Tumor Biol. 31 (2010) 363-372.

[13] J. Folkman, Self-regulation of growth in three dimensions. J. Exp. Med. 138 (1973) 745-753.

[14] H. Greenspan, Models for the growth of a solid tumor by diffusion. Stud. Appl. Math. L1 (1972) 317-340.

[15] H.P. Greenspan, On the growth and stability of cell cultures and solid tumors. J. Theor. Biol. 56 (1976) $229-242$.

[16] D.R. Grimes, C. Kelly, K. Bloch and M. Partridge, A method for estimating the oxygen consumption rate in multicellular tumour spheroids. J. Roy. Soc. Interface 11 (2014) 20131124.

[17] D. Hanahan and R.A. Weinberg, The hallmarks of cancer. Cell 100 (2000) 57-70.

[18] D. Hanahan and R.A. Weinberg, Hallmarks of cancer: the next generation. Cell 144 (2011) 646-674.

[19] F. Hirschhaeuser, H. Menne, C. Dittfeld, J. West, W. Mueller-Klieser and L.A. Kunz-Schughart, Multicellular tumor spheroids: an underestimated tool is catching up again. J. Biotechnol. 148 (2010) 3-15.

[20] M.E. Hubbard and H.M. Byrne, Multiphase modelling of vascular tumour growth in two spatial dimensions. J. Theor. Biol. 316 (2013) 70-89.

[21] K.A. Landman and C.P. Please, Tumour dynamics and necrosis: surface tension and stability. IMA J. Math. Appl. Med. Biol. 18 (2001) 131-158.

[22] G. Lemon and J.R. King, Travelling-wave behaviour in a multiphase model of a population of cells in an artificial scaffold. J. Math. Biol. 55 (2007) 449-480.

[23] G. Lemon, J.R. King, H.M. Byrne, O.E. Jensen and K.M. Shakesheff, Mathematical modelling of engineered tissue growth using a multiphase porous flow mixture theory. J. Math. Biol. 52 (2006) 571-594.

[24] R.J. LeVeque, Finite Difference Methods for Ordinary and Partial Differential Equations. Society for Industrial and Applied Mathematics (2007).

[25] T.D. Lewin, P.K. Maini, E.G. Moros, H. Enderling and H.M. Byrne, The evolution of tumour composition during fractionated radiotherapy: implications for outcome. Bull. Math. Biol. 80 (2018) 1207-1235.

[26] M. Massoudi, Boundary conditions in mixture theory and in CFD applications of higher order models. Comput. Math. Appl. 53 (2007) 156-167.

[27] R.D. O'Dea, M.R. Nelson, A.J. El Haj, S.L. Waters and H.M. Byrne, A multiscale analysis of nutrient transport and biological tissue growth in vitro. Math. Med. Biol. 32 (2015) 345-366.

[28] H. Okada and T.W. Mak, Pathways of apoptotic and non-apoptotic death in tumour cells. Nat. Rev. Cancer 4 (2004) 592-603.

[29] L. Preziosi and A. Tosin, Multiphase modelling of tumour growth and extracellular matrix interaction: mathematical tools and applications. J. Math. Biol. 58 (2009) 625-656.

[30] S. Prokopiou, E.G. Moros, J. Poleszczuk, J. Caudell, J.F. Torres-Roca, K. Latifi, J.K. Lee, R. Myerson, L.B. Harrison and $\mathrm{H}$. Enderling, A proliferation saturation index to predict radiation response and personalize radiotherapy fractionation. Radiat. Oncol. 10 (2015) 159.

[31] S. Proskuryakov and V. Gabai, Mechanisms of tumor cell necrosis. Curr. Pharm. Des. 16 (2010) 56-68.

[32] L. Tao and K.R. Rajagopal, On Boundary Conditions In Mixture Theory (1995) 130-149. 
[33] J.P. Ward and J.R. King, Mathematical modelling of avascular-tumour growth. IMA J. Math. Appl. Med. Biol. 14 (1997) 39-69.

[34] J.P. Ward and J.R. King, Mathematical modelling of avascular-tumour growth. II: Modelling growth saturation. IMA J. Math. Appl. Med. Biol. 16 (1999) 171-211.

[35] L.-B. Weiswald, D. Bellet and V. Dangles-Marie, Spherical cancer models in tumor biology. Neoplasia 17 (2015) 1-15. 